\title{
Opportunities for Discovery: Theory and Computation in Basic Energy Sciences
}

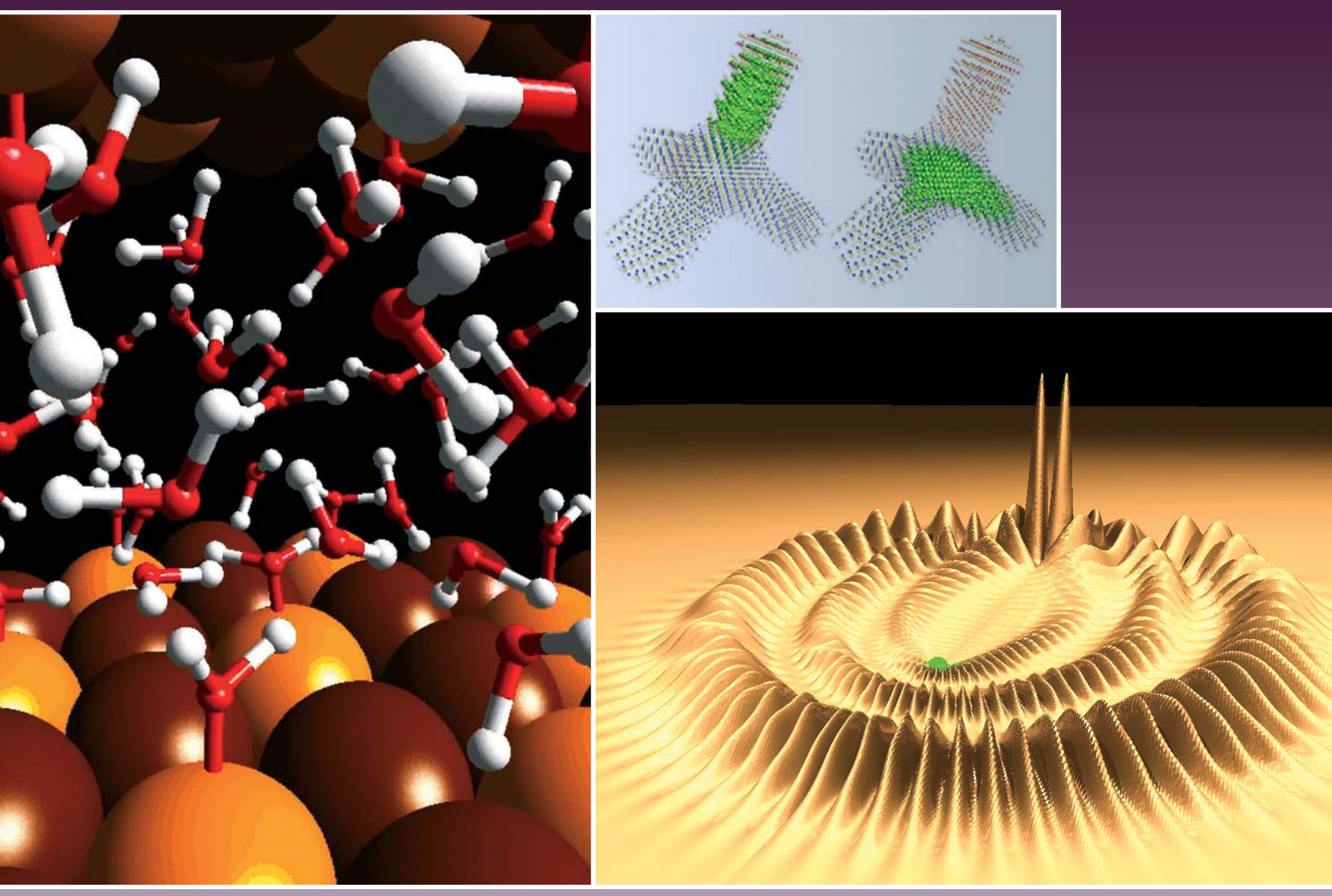

January 2005

Subcommittee on Theory and Computation of the Basic Energy Sciences Advisory Committee U.S. Department of Energy
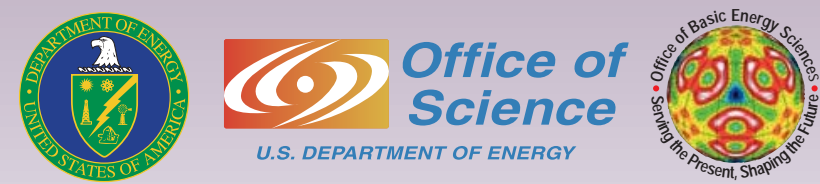
Cover images:

Front left: First-principles molecular dynamics study of the catalytic dissociation of water over a Pt-Ru catalyst, as reported in Phys. Rev. B 68, 075420 (2003). (Image courtesy of S. K. Desai and M. Neurock.)

Front upper right: Calculations of atom-by-atom electronic maps of a tetrapod with one leg of cadmium selenide and three of cadmium telluride. On the left, green marks the conduction band's lowest energy state, which is physically separated in the structure from the valence band's highest energy state, shown in green on the right. See D. J. Milliron et al., Nature 430, 190 (2004). (Image courtesy of Lawrence Berkeley National Laboratory.)

Front lower right: Electronic probability density of a rubidium Rydberg molecule that would form in an ultracold trap. This trilobite-resembling density corresponds to the equilibrium internuclear distance $R=1232$ a.u. for a highly excited perturbed hydrogenic state. The position of the $\mathrm{Rb}(5 s)$ atom is directly underneath the "twin towers" centered at $R=1232$ a.u. toward the back of the figure, while the $\mathrm{Rb}^{+}$ion is represented (with exaggerated size) as a small green sphere near the front. See C. H. Greene, A. S. Dickinson, and H. R. Sadeghpour, Phys. Rev. Lett. 85, 2458 (2000). (Image courtesy of authors.)

Back cover: A visualization of an electron in a tubular image state around a $(10,10)$ metallic carbon nanotube. Similar states should exist around any metallic or semiconducting nanotube, including multi-walled or ring-shaped nanotubes. (Reprinted with permission from B. E. Granger, P. Kral, H. R. Sadeghpour, and M. Shapiro, "Highly Extended Image States around Nanotubes," Phys. Rev. Lett. 89, 135506 (2002). Copyright 2002 by the American Physical Society.) 


\title{
OPPORTUNITIES FOR DISCOVERY: \\ THEORY AND COMPUTATION IN BASIC ENERGY SCIENCES
}

\author{
SUBCOMMITTEE ON \\ THEORY AND COMPUTATION \\ OF THE BASIC ENERGY SCIENCES \\ ADVISORY COMMITTEE \\ U.S. DEPARTMENT OF ENERGY
}

Co-chairs:

Bruce Harmon, lowa State University and Ames Laboratory

Kate Kirby, Harvard-Smithsonian Center for Astrophysics

C. William McCurdy, University of California, Davis, and Lawrence Berkeley National Laboratory

JANUARY 2005 


\title{
Subcommittee on Theory and Computation of the Basic Energy Sciences Advisory Committee
}

\author{
Roberto Car, Princeton University \\ Peter T. Cummings, Vanderbilt University \\ James Davenport, Brookhaven National Laboratory \\ Thom Dunning, University of Tennessee \\ Bruce Garrett, Pacific Northwest National Laboratory \\ Chris Greene, University of Colorado \\ Bruce Harmon, Iowa State University and Ames Laboratory \\ Rajiv Kalia, University of Southern California \\ Kate Kirby, Harvard-Smithsonian Center for Astrophysics \\ Walter Kohn, University of California, Santa Barbara \\ Carl Lineberger, University of Colorado \\ C. William McCurdy, University of California, Davis, \\ and Lawrence Berkeley National Laboratory \\ Michael Norman, Argonne National Laboratory \\ Larry Rahn, Sandia National Laboratory, Livermore \\ Anthony Rollett, Carnegie Mellon University \\ Douglas Tobias, University of California, Irvine \\ R. Stanley Williams, Hewlett Packard Laboratories \\ Margaret Wright, New York University, Courant Institute
}

Observation and theory get on best when they are mixed together, both helping one another in the pursuit of truth. It is a good rule not to put overmuch confidence in a theory until it has been confirmed by observation. I hope I shall not shock the experimental physicists too much if I add that it is also a good rule not to put overmuch confidence in the observational results that are put forward until they have been confirmed by theory.

-Sir Arthur Eddington, New Pathways in Science, 1935 


\section{Contents}

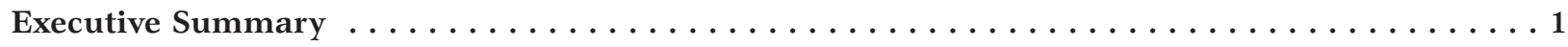

I. A Confluence of Scientific Opportunities: Why Invest Now in Theory

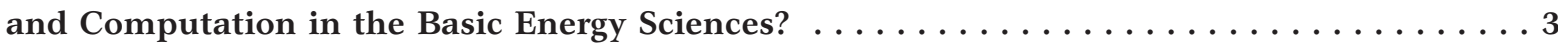

A. Dramatic Progress in Theory and Modeling in Chemistry and Materials Sciences . . . . . . . 4

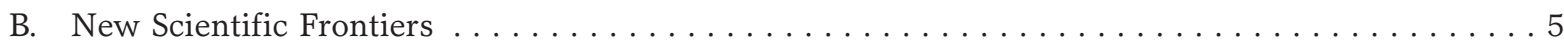

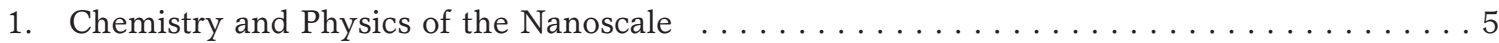

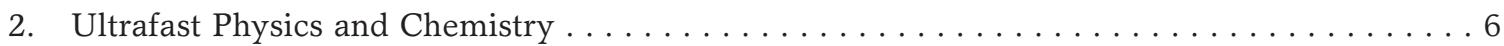

3. Control of Matter and Information at the Quantum Level $\ldots \ldots \ldots \ldots \ldots \ldots \ldots$

4. Application of Modern Biological Techniques to Chemistry

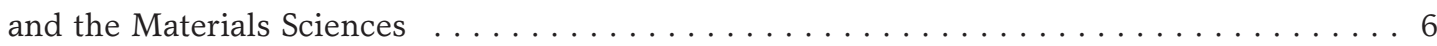

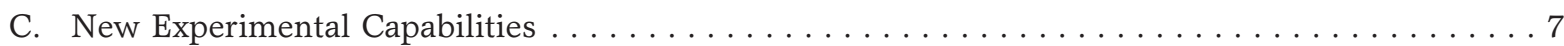

1. New Experimental Capabilities Involving "Tabletop" Science $\ldots \ldots \ldots \ldots \ldots \ldots \ldots \ldots$

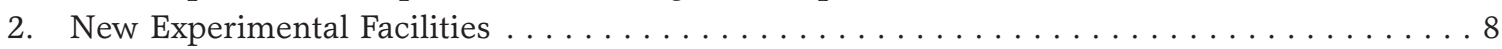

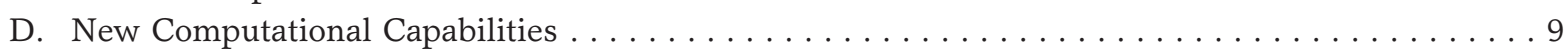

II. The Unity of Theory and Computation in the Basic Energy Sciences $\ldots \ldots \ldots \ldots \ldots \ldots$

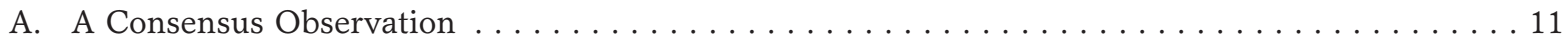

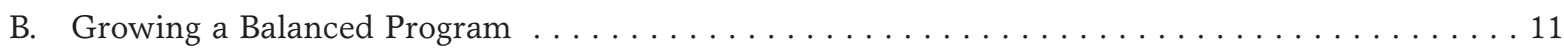

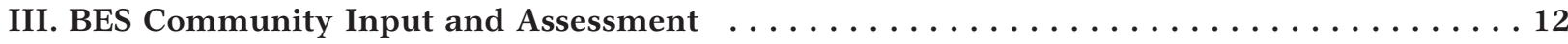

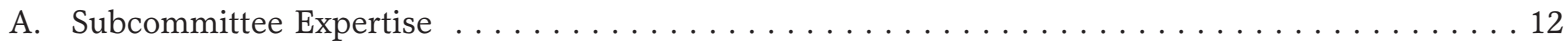

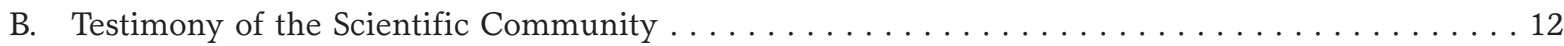

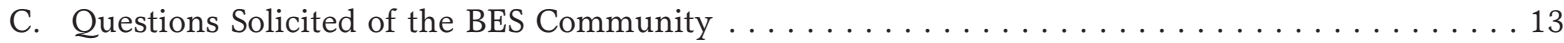

IV. Emerging Themes in BES: Complexity and Control $\ldots \ldots \ldots \ldots \ldots \ldots \ldots \ldots \ldots \ldots$

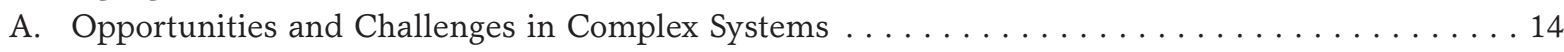

1. Nanoscience . . . . . . . . . . . . . . . . . . . . . . . . . . . . 14

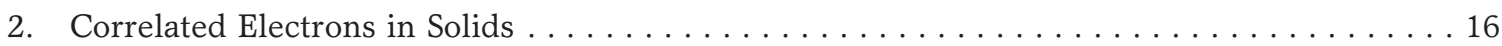

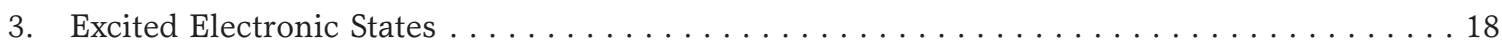

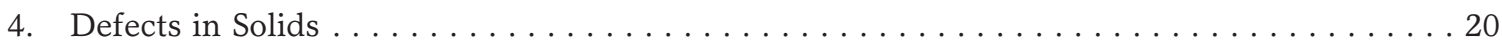

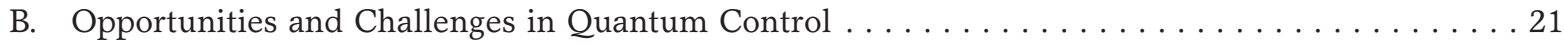

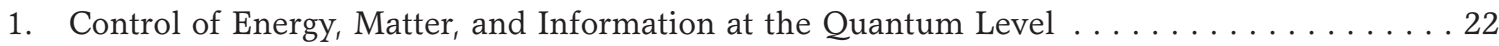

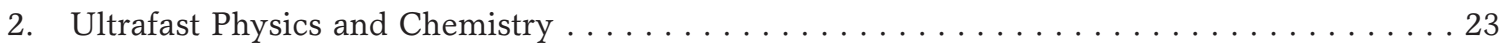

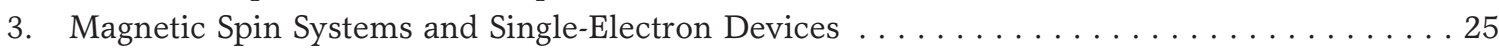

C. Opportunities and Challenges in Control of Complex Systems $\ldots \ldots \ldots \ldots \ldots \ldots \ldots \ldots \ldots$

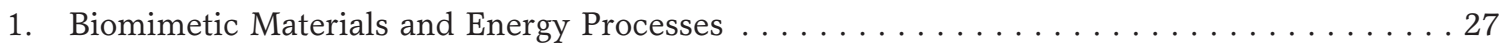

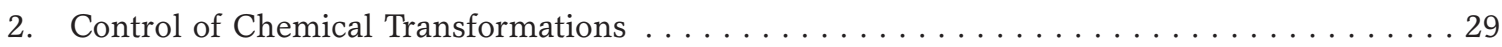


V. Connecting Theory with Experiment in BES: Accelerating Discoveries and Furthering Understanding . . . . . . . . . . . . . . . . . . . .

VI. The Resources Essential for Success in the BES Theory and Computing Enterprise . . . . . 34

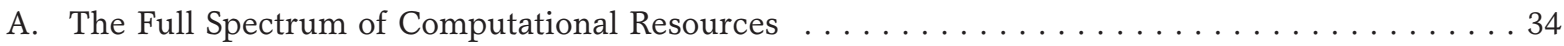

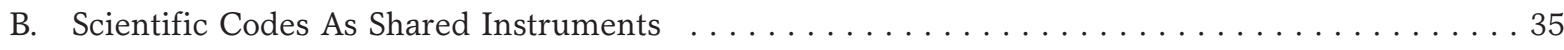

C. The Human Resources: Training Future Generations of Theorists . . . . . . . . . . . . . 37

VII. Findings and Recommendations $\ldots \ldots \ldots \ldots \ldots \ldots$

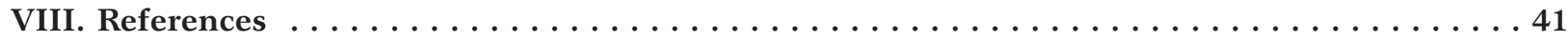




\section{Executive Summary}

New scientific frontiers, recent advances in theory, and rapid increases in computational capabilities have created compelling opportunities for theory and computation to advance the scientific mission of the Office of Basic Energy Sciences (BES). The prospects for success in the experimental programs of BES will be enhanced by pursuing these opportunities. This report makes the case for an expanded research program in theory and computation in BES.

The Subcommittee on Theory and Computation of the Basic Energy Sciences Advisory Committee was charged with identifying current and emerging challenges and opportunities for theoretical research within the scientific mission of BES, paying particular attention to how computing will be employed to enable that research. A primary purpose of the Subcommittee was to identify those investments that are necessary to ensure that theoretical research will have maximum impact in the areas of importance to BES, and to assure that BES researchers will be able to exploit the entire spectrum of computational tools, including leadership class computing facilities. The Subcommittee's Findings and Recommendations are presented in Section VII of this report.

\section{A confluence of scientific events has enhanced the importance of theory and computation in BES}

After considering both written and verbal testimony from members of the scientific community, the Subcommittee observed that a confluence of developments in scientific research over the past fifteen years has quietly revolutionized both the present role and future promise of theory and computation in the disciplines that comprise the Basic Energy Sciences. Those developments fall into four broad categories:

1. a set of striking recent scientific successes that demonstrate the increased impact of theory and computation
2. the appearance of new scientific frontiers in which innovative theory is required to lead inquiry and unravel the mysteries posed by new observations

3. the development of new experimental capabilities, including large-scale facilities, that provide challenging new data and demand both fundamental and computationally intensive theory to realize their promise

4. the ongoing increase of computational capability provided by continued improvements in computers and algorithms, which has dramatically amplified the power and applicability of theoretical research.

The sum of these events argues powerfully that now is the time for an increase in the investment by BES in theory and computation, including modeling and simulation.

\section{Emerging themes in the Basic Energy Sciences and nine specific areas of opportunity for scientific discovery}

This report identifies nine specific areas of opportunity in which expanded investment in theory and computation holds great promise to enhance discovery in the scientific mission of BES. While this list is not exhaustive, it represents a range of persuasive prospects broadly characterized by the themes of "Complexity" and "Control" that describe much of the BES portfolio. The challenges and promise of theory in each of these nine areas are described in detail.

\section{Connecting theory with experiment}

Connecting the BES theory and computation programs with experimental research taking place at existing or planned BES facilities deserves a high priority. BES should undertake a major new thrust to significantly augment its theoretical and computational programs coupled to experimental research at its major facilities. We also urge that such a new 
effort not be limited only to research at the facilities but also address the coupling of theory and computation with new capabilities involving "tabletop" experimental science as well.

The unity of modern theory and computation

For a number of the research problems in BES, we are fortunate to know the equations that must be solved. For this reason many BES disciplines are presently exploiting high-end computation and are poised to use it at the leadership scale. However, in many other areas of BES, we do not know all the equations, nor do we have all the mathematical and physical insights we need, and therefore we have not yet invented the required algorithms. In an expanded yet balanced theory effort in BES, enhancements in computation must be accompanied by enhancements in the rest of the theoretical endeavor. Conceptual theory and computation are not separate enterprises.

\section{Resources necessary for success in the BES theory enterprise}

A successful BES theory effort must provide the full spectrum of computational resources, as well as support the development and maintenance of scientific computer codes as shared scientific instruments. We find that BES is ready for and requires access to leadership-scale computing to perform calculations that cannot be done elsewhere, but also that a large amount of essential BES computation falls between the leadership and the desktop scales. Moreover, BES should provide support for the development and maintenance of shared scientific software to enhance the scientific impact of the BES-supported theory community and to remove a key obstacle to the effective exploitation of high-end computing resources and facilities.

In summary, we find that there is a compelling need for BES to expand its programs to capture opportunities created by the combination of new capabilities in theory and computation and the opening of new experimental frontiers. Providing the right resources, supporting new styles of theoretical inquiry, and building a properly balanced program are all essential for the success of an expanded effort in theory and computation. The experimental programs of BES will be enhanced by such an effort. 


\section{A Confluence of Scientific Opportunities: Why Invest Now in Theory and Computation in the Basic Energy Sciences?}

Scientific and technological advances in the past decade and a half have transformed the impact and importance of theory and computation in the Basic Energy Sciences. It has become clear that taking advantage of newly emerging opportunities in theory and computation will increase the pace of discovery across the entire scientific scope of the Basic Energy Sciences (BES) portfolio.

New opportunities have been created by a confluence of four broad categories of events (Figure 1) that we will discuss further below:

- striking recent scientific successes of theory and modeling,

- the appearance of specific new scientific frontiers,

- the construction of new experimental facilities and the development of new small-scale experimental capabilities, and

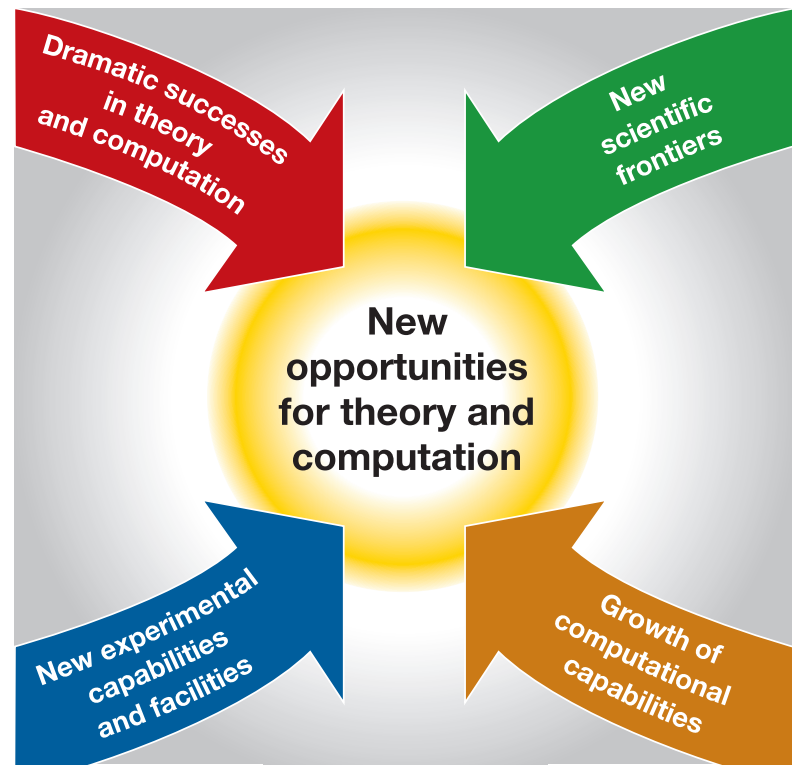

Figure 1. A confluence of scientific events has created new opportunities for theory and computation.
- the continuing growth of computational capability, including the promise of new leadership-scale computational facilities.

The ramifications of these developments are only now becoming apparent, in part because the changes happened quickly and quietly, and in part because they have occurred in a number of diverse disciplines rather than in a single area of research. The sum of these events has changed both the present reality and the future promise of theory and modeling in areas ranging from nanoscience, to spintronics, to the science of ultrafast processes in chemistry and physics.

The significance of these changes is amplified by the fact that the theory enterprise in the Basic Energy Sciences is characterized to a remarkable degree by a common basis of theoretical, mathematical, and computational tools, in spite of the wide range of subjects it spans. For example, classical or $a b$ initio molecular dynamics methods are as likely to be used in the study of liquids as in the exploration of the properties of carbon nanotubes. We find density functional methods being used in the study of large isolated molecules in the gas phase as well as in the study of the spin dynamics of magnetic ions in computer disk read heads. The fact that theoreticians drawn today from seemingly distant scientific disciplines will identify common sources of new advances that increased the impact of theory in their own research areas is evidence of the broad and fundamental importance of these basic theoretical, mathematical, and computational innovations. That commonality of basic tools also suggests the breadth of the impact that can be expected from increased investment in the theory and computation enterprise in Basic Energy Sciences.

Across the spectrum of disciplines represented in the Basic Energy Sciences, there is also ample 
evidence of extensive use of computing at all scales. It consumes a large fraction of the computer time at both Department of Energy and National Science Foundation supported high performance computing facilities. Not only are these disciplines characterized by a knowledge of the equations describing many of their problems, but also a number of large-scale codes are already written and running efficiently in production mode. The community in most of these disciplines is ready to make use of leadership-class computing, and in many cases is severely limited by the lack of access to adequate computing resources.

The new importance of theory and computation in each of the scientific areas we will discuss in this report matches new opportunities for discovery and exploration at the heart of the mission of the Office of Science. The reason to invest wisely now in theory and computation in the Basic Energy Sciences is that a combination of recent events has dramatically increased the likely scientific yield that will result from that investment. A sea change is under way for the theory and computation enterprise, and the specific opportunities it brings may be missed without the prompt attention of the Office of Basic Energy Sciences.

\section{A. DRAMATIC PROGRESS IN THEORY AND MODELING IN CHEMISTRY AND MATERIALS SCIENCES}

New experimental facilities and capabilities are uncovering a wealth of problems requiring new theoretical concepts and approaches. Breakthrough conceptual ideas are critical for solving such problems, and they often lead to novel algorithms and effective computational approaches to understanding, control, and further exploration. Throughout the Office of Basic Energy Sciences, there are numerous examples of striking progress on such key scientific problems. In many of these subjects at present, "the equations are known," and it is in the method of their solution that great advances with far-reaching consequences have been made. An abbreviated list of those examples paints a vivid picture of their broad impact:

- Density functional theory (DFT), the conceptual development of which was recog- nized by the 1998 Nobel prize, has transformed theoretical chemistry, surface science, and materials physics. When coupled with modern computational tools, DFT has created the ability to describe the ground state electronic structure, mechanical and optical properties, and interatomic forces in systems containing hundreds and sometimes thousands of atoms.

- Large-scale classical molecular dynamics, augmented by fast multipole methods for computing long-range interatomic forces, has matured to make calculations involving the motion of hundreds of thousands to millions of atoms fairly routine, and simulations involving as many as billions of atoms have been reported in recent years. It has also been implemented in extensive publicly available computer codes that perform well on parallel computers (such as LAMMPS and NAMD), and in widely used codes like AMBER and CHARMM that are either publicly or commercially available.

- Discrete grid and wave packet methods for treating the motion of electrons and nuclei in atomic and molecular physics have made it possible to treat atoms and molecules in intense or short-pulsed laser fields and opened the door to the description of ultrafast processes, including those that involve electronic changes approaching the attosecond time scale.

- Ab initio molecular dynamics simultaneously calculates the motion of atoms and the accompanying changes in electronic structure, making possible the treatment of processes in condensed phase systems in which the bonding between atoms changes. This development has brought new problems like the elasticity of minerals and the properties of metals and semiconductors in disordered or liquid states within the range of accurate calculations.

- A range of improved approaches, including coupled cluster, many-body perturbation theory, and quantum Monte Carlo methods, have evolved strikingly over the last fifteen years to make possible accurate electronic structure calculations of the properties of 
chemically important molecules ( $\leq$ about 20 atoms) and prototypical solids.

- First-principles spin dynamics elucidated the mechanism of giant magnetoresistance, which underlies modern data storage systems, and opened the door to predicting other properties of spin (electron) transport in these systems and thus to other applications in the new field of "spintronics."

- Dynamical mean field theory (DMFT) can now go beyond band theory and simple models to evaluate the electronic structure of strongly correlated electronic states in solids. For transition metal oxides, rare earths, and many actinide materials, density functional theory using the local density approximation (LDA) fails, whereas DMFT has proven successful by self-consistently combining elements of band theory with local on-site Coulomb correlations to explain such outstanding phenomena as metal-insulator transitions, unusual charge and spin orderings in cuprates, and dramatic responsiveness of narrow band materials (e.g., plutonium) to pressure and temperature changes.

This list can be extended to include many more examples of a stunning array of new theoretical capabilities in chemistry, materials sciences, and atomic and molecular physics, some of which have come about as a result of completely new approaches, and others from decades of small improvements that brought them to maturity. They paint a portrait of a much more robust theoretical and computational enterprise than existed fifteen years ago.

These advances also depict an enterprise ready to exploit high-end parallel computing. In many of these disciplines and applications we already know the equations we must solve. Many of the important algorithms for solving them are known to adapt easily to large-scale parallelism. Molecular dynamics, quantum Monte Carlo methods, density functional theory, and classical Monte Carlo simulations exploit those algorithms. Computer codes that implement them on terascale computers already exist and are in common use in quantum chemistry and materials science to investigate electronic structure and atomic scale motion in scores of important applications.
The advances listed here, together with many others, form the basis of the current use and the future promise of high-end computing in the Basic Energy Sciences. Further evidence of the increasing impact of BES programs in theory and computation is provided by the tremendous growth of the number of interdisciplinary practitioners of these new methods in engineering, biological, and industrial departments, where the basic science is being coupled with modern computational facilities to pursue problems of great complexity and significance.

\section{B. NEW SCIENTIFIC FRONTIERS}

Over the last decade several new scientific areas have begun to take shape as major thrusts in chemistry and the materials sciences. In almost all cases those areas have grown out of the success of innovative experimental techniques that opened new horizons. Sometimes the subjects have existed for decades but have been revitalized by unexpected discoveries or advanced experimental methods. In all of these cases the consequence has been to produce a wealth of challenges for the theory community and a heightened priority for innovative theory to guide and interpret experiments. The emergence of these frontiers forms an essential part of the context of this report. Several, but not all, of the new scientific opportunities identified in later sections and in the findings and recommendations derive from these broad new directions in twenty-first century science.

\section{Chemistry and Physics of the Nanoscale}

A new set of techniques, such as atomic force microscopy and scanning tunneling microscopy, catalyzed the revolution that has been identified by the scientific community as a new priority in the National Nanotechnology Initiative [1]. Nanoscale systems are the smallest systems that exhibit multiple length and time scales - from atoms $(0.1 \mathrm{~nm})$ to macromolecular assemblies (100 nm or larger), and from atomic motions (femtoseconds) to protein folding (milliseconds or longer). A central computational challenge of nanoscience is bridging length and time scales so that phenomena captured in atomistic simulations can be applied at the nanoscale. Stunning successes are guiding and 
interpreting experiments in this area already, such as the calculation of the electronic properties of nanotubes and the electronic structure of thousandatom tetrapods with one leg of cadmium selenide and three of cadmium telluride. Other aspects of nanoscience, such as the prediction of self-assembling structures, will require entirely new theory. A set of the challenges and opportunities for theory in this area was already summarized in the recent BESAC report entitled "Theory and Modeling in Nanoscience," which can be found at http://www.sc.doe.gov/bes/reports/list.html.

\section{Ultrafast Physics and Chemistry}

The field of ultrafast science emerged from the development of lasers capable of producing femtosecond pulses. The 1999 Nobel Prize in Chemistry was awarded for the investigation of chemical reactions, including the probing of shortlived intermediate complexes. The advent of laser pulses of a few hundred attoseconds is opening the way to probing events on the time scale of electronic motions, such as changes in electronic structure during an Auger decay of an atom or molecule. Many of the theoretical techniques needed to accurately describe events on these time scales already exist, and numerical wave packet solutions of the time-dependent Schrödinger equation have become common both for nuclear and electronic motions in small systems. In this area there is tremendous promise for theory coupled with highend computation to guide the design of new experiments and new experimental facilities. There are also great challenges. Modern femtosecond experiments can involve electronically excited states of complex molecules with intersecting potential surfaces, and the description of attosecond experiments will require the time-dependent treatment of many-electron systems. Great scientific payoffs can certainly result from an enhanced theory effort.

\section{Control of Matter and Information at the Quantum Level}

A broad array of contemporary scientific thrusts involves the manipulation of matter or information at the quantum level and the corresponding pursuit of its potential applications in new technologies. Diverse examples reveal this unifying theme: (A) With new experimental techniques probing atoms, clusters, and dots at the nanoscale, creating and controlling quantum states has become a laboratory frontier that may ultimately produce quantum computers. (B) The entirely new discipline of "spintronics" has emerged from new experimental capabilities. (C) Since the creation of the first BoseEinstein condensate from dilute, ultracold samples of alkali atoms, the field of ultracold physics and the study of trapping and cooling gases to reach quantum degeneracy has burgeoned. (D) Using intense, shaped laser pulses it is possible to direct a quantum system into a desired physical state and thereby induce molecules to dissociate preferentially to produce different products, depending on the shape and phase of the controlling laser pulses. All of these examples of manipulation of matter at the quantum level offer dramatic opportunities for the partnership of experiment with new computationally intensive theory. For example, accurate portrayal of the evolution of quantum states, including interactions with realistic environmental baths, will require following the time development of millions of coupled states for long times compared to the decoherence time - a formidable but essential computational task.

\section{Application of Modern Biological Techniques to Chemistry and the Materials Sciences}

The dramatic growth of new capabilities in molecular biology and biochemistry has revealed extraordinary sources of new materials. Interdisciplinary teams are studying how to adapt, mimic, and modify biological processes for synthesis routes leading to materials suitable for uses of broad technological interest. Photosynthetic processes may lead to hydrogen production, while membrane modifications may lead to filters for separating toxic wastes. Lightweight structural materials which self-assemble outside of living systems, or molecular motors that can be incorporated on chips at the nanoscale are not just science fiction anymore. Biology in general has not had the tradition of a strong partnership with theory and computation, but the situation is rapidly changing as the exciting possibilities and outstanding problems are attracting many talented young scientists. Genetics, protein folding, membrane mechanics, and self-organization are just a few of the topics representing complex biological materials and phenomena that are starting 
to benefit from the attention of interdisciplinary teams which include strong theory and computational components (see the recent report "The Role of Theory in Biological Physics and Materials" [2]).

This list of new scientific frontiers does not by any means exhaust the list of major new directions in science in this century nor does it completely represent the list of disciplines in which we have identified new opportunities for theory and computation in the Basic Energy Sciences. However, these major new scientific trends emerged repeatedly in the testimony given to this Subcommittee by the BES community, and they signal the excitement that characterizes many of the new directions that theory and computation in the Basic Energy Sciences may take.

\section{NEW EXPERIMENTAL CAPABILITIES}

A significant portion of the need for increased coupling between theory and experiment derives from the development of qualitatively new tools and capabilities by experimentalists, enabling the quantitative investigation of well-characterized targets, with specificity and control almost unimagined a few years ago. However, one outcome of the increased complexity is that the observed signals rarely contain all the information required for complete understanding of the results. As a consequence, theory, computation, and modeling have become an essential component of the majority of the new state-of-the-art experimental programs. While this coupling has of course occurred in the past, it has never been as essential as it is now. This fact is readily apparent in the rapidly increasing number of publications jointly authored by theorists and experimentalists.

These new experimental capabilities have come from innovation both at the individual investigator level and in the new facilities constructed by DOE. Several of these exciting capabilities are highlighted below, in the context of the science drivers summarized in the preceding section.

\section{New Experimental Capabilities Involving "Tabletop" Science}

Laboratory-based experiments at universities, the national laboratories, and even some industrial laboratories have continued to be a major source of innovation and fundamental breakthrough science in the United States. These efforts involve both single- and multiple-PI efforts, most often carried out by graduate students and postdoctoral researchers. Some, although not all, such experiments involve the invention and use of new classes of laboratory-based instruments. These small-scale experimental efforts often lead to new classes of experiments at the large national facilities. A few examples in which the results of laboratory-based experiments have had major impacts during the last decade are listed below.

The field of ultrafast science evolved from the development of femtosecond tabletop laser sources, capable of providing experimental observations of molecular properties on the time scale of nuclear motions. The development of sophisticated pulseshaping techniques holds the exciting promise of providing a degree of control over the temporal evolution of systems with moderate complexity. New ultrafast experiments include pulsed electron diffraction experiments and optical high harmonic generation, providing ultrafast soft $\mathrm{x}$-rays that have been used for photoelectron spectroscopy experiments. Both tabletop and accelerator-based x-ray sources are being developed to provide increased time resolution for the next generation of experiments.

Major advances in imaging spectroscopies have provided researchers with the ability to study individual molecules in great detail. In the early 1980s, the implementation of probe microscopies enabled the direct visualization of atoms and molecules on surfaces, with atomic resolution. No information, however, was provided on the identity of the species imaged. A major breakthrough occurred with the demonstration of single-molecule vibrational spectroscopy through the observation of inelastic tunneling of electrons in an STM experiment (a BES-funded study). Vibrational spectroscopy of the imaged molecule provides molecular identification. Nuclear magnetic resonance (NMR) is even now being coupled with probe microscopies to provide nanometer spatially resolved spectroscopies.

The ability to cool atoms in laser-based atom traps led to the production of ultracold, dilute gas Bose-Einstein condensates in the laboratory. Experiments on ultracold atoms have spawned 
completely new areas of research and are enabling the study of many fundamental questions, such as quantum coherence. The role of theory in this enterprise is especially crucial, as many of the concepts are not well formulated, and the models for success in this area all involve close experimental/ theoretical collaborations.

In other areas as well, "tabletop" experiments are making remarkable progress. The study of liquid interfaces has been enhanced by the increased availability of lab-based picosecond and femtosecond pulsed lasers, while in synthesis major advances include the application of self-assembly techniques, the development of nanometer structured materials, and the creation of biomimetic materials with complex pore shapes and sizes.

All of the examples mentioned above are at stages where strong coupling of the experimental efforts with theory and computation is essential.

Altogether new theoretical approaches are urgently needed to understand ultrafast chemistry and physics, particularly at the attosecond scale, where electronic motion and transitions are probed directly. Fundamental questions concerning quantum coherence as well as the nature of interactions of cold atoms must be answered to exploit the new class of experiments made possible by laser cooling and trapping. We do not yet have an understanding of the selection rules that govern vibrational inelastic tunneling spectroscopy in a scanning tunneling microscopy experiment. The understanding of the interfaces of liquids and solutions will require new advances in simulation methods. Even novel methods of synthesis require new theoretical approaches to guide the application of self-assembly to ever more complex systems.

\section{New Experimental Facilities}

The past decade and a half has seen the construction of new experimental facilities funded by the Office of Basic Energy Sciences and the expansion of others. Those facilities came into being in response to the needs of the scientific community. After their construction, the facilities sometimes opened new frontiers in areas that were barely contemplated when they were conceived.

During the past fifteen years the class of facilities that are called collectively the "light sources," producing intense $\mathrm{x}$-ray radiation for a wide range of experiments, has been dramatically expanded. In
1990 the construction of the Advanced Photon Source at the Argonne National Laboratory was begun. In 1999 upgrades were initiated of both the Advanced Light Source at the Lawrence Berkeley National Laboratory and the National Synchrotron Light Source at the Brookhaven National Laboratory. A new light source, the Linac Coherent Light Source, is under construction at the Stanford Linear Accelerator Center. There were some unexpected consequences of the existence of the light sources, such as their central role in high-throughput protein crystallography in the life sciences. These facilities are also fulfilling the purposes for which they were conceived. They have created a growing wave of new experiments in chemistry, physics, and the materials sciences, and have thereby challenged theory in an array of disciplines and created a need for expanded theoretical research to meet those challenges.

Construction of the Spallation Neutron Source (SNS) at the Oak Ridge National Laboratory began in 1999 and is scheduled for completion in 2006. The beamlines and experimental stations planned for this facility will be able to probe a wide range of length scales $\left(0.1\right.$ to $\left.10^{5} \AA\right)$ as well as a wide range of atomic and magnetic excitations (1 to $10^{-7} \mathrm{eV}$ ). New challenges to theory are expected in many areas of materials science, ranging from the properties of superconductors to polymer dynamics. Theoretical research in areas that will support and guide experiments at the SNS is urgently needed.

Five Nanoscale Science Research Centers are under design or construction: the Center for Nanophase Materials Sciences at Oak Ridge National Laboratory, the Molecular Foundry at Lawrence Berkeley National Laboratory, the Center for Integrated Nanotechnologies at Sandia National Laboratory, the Center for Functional Nanomaterials at Brookhaven National Laboratory, and the Center for Nanoscale Materials at Argonne National Laboratory. Collectively they represent a new kind of national facility, each of which assembles a wide range of new capabilities for research in nanoscience and also exploits the neutron and light sources. These facilities are expected to change the face of research in nanoscience. Their activities are intensifying the need for expanded theoretical efforts in nanoscience, particularly theory associated with the experiments that will be performed at them.

The growth over the past decade and a half in 
the number and type of experimental facilities constructed by the Office of Basic Energy Sciences represents a well-planned and executed expansion of experimental activities to the scale of hundreds of millions of dollars per year. The planning and design of these facilities were undertaken with a view to balancing the entire portfolio of experimental areas represented in BES. It is striking that there has been no overall plan for an accompanying expansion of theoretical research in these areas, let alone much of an increase in the support of that research, even though it is obvious that both are urgently needed.

\section{NEW COMPUTATIONAL CAPABILITIES}

The emergence of new computational capabilities has played a central role in the dramatic progress seen in theoretical research in the Basic Energy Sciences. Those capabilities fall broadly into two classes: (1) workstation and cluster computing in the laboratory, and (2) massively parallel computing at major computational facilities.

The rapid growth in the power of computers with each passing year has come to be expected by everyone who uses them. Scientists are fond of referring to this technological phenomenon as Moore's Law, referring to Gordon Moore's observation that the power of microprocessors has long been doubling every 18 months. Another equally striking aspect of the phenomenon is that the price of those microprocessors continues to decline rapidly. This predictable trend has been exploited to great advantage by investigators in the Basic Energy Sciences. It has allowed the development of many of the methods that led to dramatic advances in the power of computationally intensive theory that are discussed in this report.

The idea of a "cluster computer," referring to a collection of tens or hundreds of processors that can be assembled or purchased today by a single research group or department, has become a widespread strategy for providing computing resources in universities and national laboratories. The point that is central to this report is that this class of computing has been exploited by researchers in the Basic Energy Sciences in a way that has quietly revolutionized the underlying computational meth- ods of our collective disciplines. Clusters have been the springboard for launching the parallel computing algorithms and implementations that have readied those disciplines for massively parallel computing. This quiet transformation is credited with amplifying the impact of modern quantum chemistry by making it accessible to experimentalists as well as theoreticians. In materials science, it was exploited by the BES project known as the Computational Materials Science Network to seed the development of community codes in the materials sciences. Other examples abound in the BES portfolio. They are a significant part of the evidence that these communities are capable of exploiting larger computing resources.

Large-scale computing facilities in this country are operated by the Department of Energy and the National Science Foundation as well as by individual states and universities with funding from a variety of sources. Computationally intensive research in the Basic Energy Sciences is carried out at almost all of these facilities. Work in these disciplines is frequently the subject of highlights from these facilities. Over the last fifteen years the Department of Energy has continually upgraded its National Energy Research Scientific Computing Center and other centers at the Oak Ridge and Argonne National Laboratories. The DOE has created a new computing facility at the Environmental Molecular Sciences Laboratory at the Pacific Northwest National Laboratory, and has begun the creation of a new leadership-class computing facility at Oak Ridge.

BES researchers are major users of the facilities listed above. Research in the Basic Energy Sciences forms around $20 \%$ of the use of the National Energy Research Scientific Computing Center. One of only three initial awards of millions of hours of computer time in the DOE program known as Innovative and Novel Computational Impact on Theory and Experiment (INCITE) went to a project in computational chemistry making use of quantum Monte Carlo techniques. That award also emphasizes the point that the computational efficiency on massively parallel computers of codes that make use of many of the standard methods in these disciplines is exceptionally high. Examples include codes implementing classical molecular dynamics, classical and quantum Monte Carlo methods, and density functional based spin 
dynamics. A second INCITE award of 2.5 million processor hours was given this year to BES researchers for numerical simulation of turbulent non-premixed combustion. Chemistry and materials sciences were a major part of the proposal for the leadership-class facility at Oak Ridge.

The DOE's investments in its computing facilities, especially the most recent ones to establish leadership-class facilities, represent a tremendous opportunity and stimulus for theory in the Basic Energy Sciences, and one for which it has amply demonstrated that it is ready. Nevertheless, as we will discuss below, insufficient access to high-end computational facilities for the BES research portfolio remains a barrier to future progress. A hierarchy of hardware resources is required to meet the needs of that community, and we will discuss the requirements of the BES theory enterprise for all levels of that hierarchy. 


\section{The Unity of Theory and Computation in the Basic Energy Sciences}

\section{A. A CONSENSUS OBSERVATION}

In the view of the Subcommittee, BES theory and computation should be considered as a unity, and not as competing parts of the BES portfolio. By "computation" we do not mean simply raw computing power; we mean the deliberately integrated combination of mathematical modeling and analysis, creation of numerical algorithms, software implementation, and visualization of computed results. We also stress that there is often no clear distinction between conceptual theory and the formulation of mathematical models.

The theory enterprise within the fields of science supported by the Office of Basic Energy Sciences of DOE is heterogeneous, and properly so, not only with respect to the scientific problems being addressed, but also with respect to research group size and computational resources required. Innovation flourishes at the single-PI level, providing new theories and insights, the development of new computational methods, and the education and training of future theorists. At the other end of the spectrum is a new mode of theory and computation, often involving large multidisciplinary groups, investigating problems of great complexity. The solution of such problems requires the development and utilization of large-scale computer codes that demand leadership-class computer hardware, and the implementation of new mathematical methods designed to make efficient use of the hardware.

A consensus has emerged in the Subcommittee, and among the researchers who provided testimony, that in order to ensure the highest quality scientific return, the wisest investment on the part of the Office of Basic Energy Sciences is to support the complete spectrum of theory activity, in a thoughtful and balanced way, and to provide access to the full range of computational resources appro- priate to the particular research being pursued. In each of the areas of new scientific opportunity for theory and computation identified in the following section, it will be important to avoid making investment decisions that effectively replace one key part of the theory enterprise with another.

\section{B. GROWING A BALANCED PROGRAM}

This report recommends an increase in BES-funded efforts in theory and computation across its portfolio. In subsequent sections specific areas of opportunity are discussed and arguments provided for new investment in them. The input of the research community and the Subcommittee deliberations that led to those recommendations frequently returned to the theme of how these new activities should be balanced. The total budget for DOE facilities that are funded through BES has grown dramatically over the past decade, but the core research programs in BES have seen no such growth, and therefore the overall buying power of those programs has decreased. Nonetheless, one of the arguments for growth of BES efforts in theory and computation concerns the connection of those core efforts in theory and computation with experimental research at the facilities.

A successful program will require that a balance be established between the efforts in the core program and those associated with the facilities. It will also require attention to the balance between single-PI and collaborative research as well as between conceptual theory and computationally intensive research as discussed above. Finally, a balance of investments in universities and national laboratories needs to consider such factors as training needs, effective group sizes, and connection to experimental driving forces. 


\section{BES Community Input and Assessment}

\section{A. SUBCOMMITTEE EXPERTISE}

The Subcommittee on Theory and Computation consists of 18 individuals from a mix of research universities, DOE laboratories, and industry. Approximately half of the subcommittee is in the area of materials science, and half in chemical science. Most members are theorists and/or computational chemists/physicists, although two members (Lineberger and Williams) are from the experimental community. A number of Subcommittee members have at some point in their careers been actively involved in developing computational methods to address problems in chemical and materials science.

The Subcommittee is listed below:

- Roberto Car, Princeton University, rcar@princeton.edu

- Peter T. Cummings, Vanderbilt University, peter.cummings@vanderbilt.edu

- James Davenport, Brookhaven National Laboratory, jdaven@bnl.gov

- Thom Dunning, University of Tennessee, dunningthjr@ornl.gov

- Bruce Garrett, Pacific Northwest National Laboratory, bruce.garrett@pnl.gov

- Chris Greene, University of Colorado, Chris.Greene@Colorado.edu

- Bruce Harmon, Iowa State University and Ames Laboratory, harmon@ameslab.gov

- Rajiv Kalia, University of Southern California,rkalia@usc.edu

- Kate Kirby, Harvard-Smithsonian Center for Astrophysics,kirby@cfa.harvard.edu

- Walter Kohn, University of California, Santa Barbara,kohn@physics.ucsb.edu

- Carl Lineberger, University of Colorado, wcl@jila.colorado.edu

- C. William McCurdy, University of California, Davis, and Lawrence Berkeley National Laboratory, CWMcCurdy@lbl.gov
- Michael Norman, Argonne National Laboratory,norman@anl.gov

- Larry Rahn, Sandia National Laboratory, Livermore,rahn@sandia.gov

- Anthony Rollett, Carnegie Mellon University, rollett@andrew.cmu.edu

- Douglas Tobias, University of California, Irvine,dtobias@uci.edu

- R. Stanley Williams, Hewlett Packard Laboratories,Stan_Williams@hp.com

- Margaret Wright, New York University, Courant Institute,mhw@cs.nyu.edu

The first meeting of the Subcommittee took place February 22, 2004, one day prior to the BESAC meeting in Washington, D.C. A second meeting, described below, was held in April for the purpose of taking verbal testimony from the community and discussing the issues that the Subcommittee would address. A preliminary "letter report" arising from these meetings was formally transmitted to BESAC at its meeting in August 2004. Finally, on October 9, 2004, a meeting was held in Denver to finalize the findings and recommendations of the Subcommittee that are contained in Section VII.

\section{B. TESTIMONY OF THE SCIENTIFIC COMMUNITY}

In order to obtain input that would inform our assessment of specific new opportunities, the Subcommittee organized a meeting April 17 and 18, 2004 in a hotel near O'Hare Airport to take testimony from the scientific community. The Subcommittee pooled suggestions of individuals across a broad range of BES science who were invited to articulate, in person, the case for opportunities in various scientific areas and to opine on the needs for theory and computation support generally. The ques- 
tions developed by the Subcommittee which these individuals were asked to address are listed in the next section.

The Subcommittee solicited input generally from both the chemistry and materials science communities by establishing a website at https://besac.nersc.gov and sending out mass emails inviting testimony to members of the American Physical Society in the following divisions: Atomic, Molecular and Optical Physics, Chemical Physics, Materials Physics, and Condensed Matter Physics. In addition, an announcement of this opportunity to provide input was posted prominently on the American Chemical Society Division of Physical Chemistry home page (http://hackberry.chem. trinity.edu/PHYS//). Over 44 scientists submitted testimony to the website.

\section{QUESTIONS SOLICITED OF THE BES COMMUNITY}

1. In your field, what are the major scientific challenges? What principal opportunities for discovery and progress are appearing? And why? What explicit important problems can be addressed by theory and/or computation? Your reply will be most useful if the science it describes is both understandable and compelling to a wide scientific audience.

2. In your area, does theory and computational science drive progress and/or have a mean- ingful partnership with experiment in pushing discoveries? If not, what are the barriers?

3. How might progress in your field impact other areas, particularly those within the purview of the DOE Office of Basic Energy Sciences? If possible, relate the major challenges and opportunities you have articulated to the experimental programs or facilities funded by BES.

4. Are computing resources (hardware and software) a limiting factor in your field? Could an increase in computational power by two or three orders of magnitude make a major difference? What new computational resources would your own research most benefit from in the next two to five years?

5. Would support for development of new algorithms for high-end computer architectures and for maintaining large suites of codes be important in making progress in your broad community? If you use software written by others, does it meet your needs?

6. Are there opportunities for colleagues in your area to form partnerships with scientists in related fields in order to assemble interdisciplinary teams for attacking important overarching problems? If so, is there presently software spanning the related disciplines? Or would the necessary software need to be assembled or developed as part of the agenda to advance the science? 


\section{Emerging Themes in BES: Complexity and Control}

Research in the BES portfolio is richly diverse, impacting a broad spectrum of energy initiatives and DOE priorities. Despite this diversity, which is one of the portfolio's greatest strengths, it is possible to identify two over-arching themes characterizing forefront BES science: the treatment of ever more complex systems, and the control of atomic and molecular interactions and processes at the quantum level. An ultimate goal is the union of these two themes, such that control of complex systems can be exploited in the development of novel energy technologies, new methods of treating radioactive waste, and innovative applications of fundamental chemistry and physics impacting such areas as computer design, bioinspired materials, and environmental remediation.

\section{A. OPPORTUNITIES AND CHALLENGES IN COMPLEX SYSTEMS}

Understanding real systems demands methods for treating structure and dynamics taking place on surfaces, in liquids, and in disordered media. For the past forty years, theoreticians developing quantum chemistry and electronic structure codes have strived to treat systems with increasing numbers of electrons, and molecules (or unit cells) with greater numbers of atoms, at ever higher levels of accuracy. Innovative methods to study such systems with reasonable accuracy have demanded the highest level of computational capability available. Despite the many advances in techniques and algorithms that have been implemented over the last two decades, it is still true that progress in characterizing complex molecules and solids is intimately coupled to the availability of high-end computer facilities.

In many cases quantitative models governing the interactions of complex systems are not yet formulated, and simulations demanding extensive computational capabilities are the first line of approach to understanding experiments and to developing constitutive equations with predictive capability.

Treating processes taking place on multiple length and time scales continues to challenge theorists. Opportunities for discovery in the BES portfolio which fall in the "complex systems" category include nanoscience, correlated electrons in solids, excited electronic states, and defects in solids, all of which are discussed below.

\section{Nanoscience}

Unprecedentedly small and highly functional medical and computational devices are just a few of the promises of nanoscience and nanotechnology which have captured the imagination of researchers and the attention of funding agencies. The U.S. annual federal investment in nanoscience is now reaching $\$ 1 \mathrm{~B} / \mathrm{yr}$. Similar excitement and investments have emerged in Europe, Japan, and elsewhere. While the promises are enormous, the challenges facing nanoscience researchers are formidable.

A critical area that must be developed for success in nanoscience is the use of scanning probes for imaging, characterization and manipulation of materials on the nanoscale. Scanning probes place demands on theory and computation ranging from the development of continuum theory algorithms, to first principles calculations of images and vibrational spectra (see Figure 2). Theoretical insights for enhanced light scattering at probe tips are needed, as are designs for absolutely new modes for scanning.

Another key to success for nanoscience is the manufacture of nanostructured materials by selfassembly, which requires fundamental understanding of the connection between microscopic behavior and macroscale processing variables (tempera- 


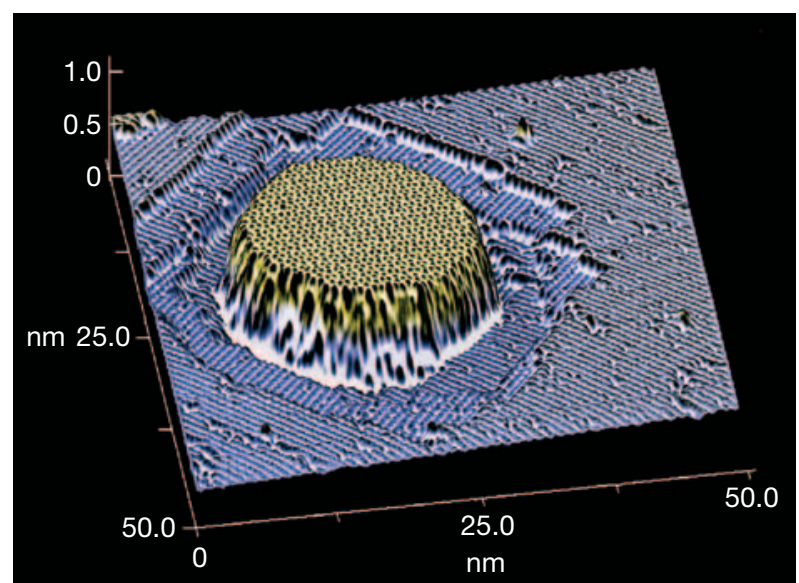

Figure 2. A scanning tunneling microscope image of a self-assembled titanium disilicide island on a $\mathrm{Si}(100)$ surface. These islands act as seeds for the catalytic growth of silicon nanowires. This high-resolution image was collected in 1999, but the detailed atomic structure of the top surface of the silicide island is still not understood, because the electronic structure and the locations of the atoms are convoluted in the image, and only a significant theory and computational effort can deconvolute them. (Image courtesy of G. MedeirosRibeiro, Hewlett-Packard Labs.)

ture, pressure, exposure time, concentration, etc.) to achieve high reproducibility and product quality. The understanding and control of the complex forces and processes encountered at the nanometer scale will require an intimate coupling between theory, computation, and experiment. Indeed, the necessity of this partnership has been recognized from the beginning by the National Nanotechnology Initiative (NNI) $[1,3]$. This recognition is also evident at the new nanoscale science research centers being established as user facilities at five DOE national laboratories, where each is being constituted with an in-house theory and computation group.

One example in which the interplay between theory and experiment is in full force is in the determination of the conductance properties of a single molecule. Measurements by Reed and coworkers in 1997 [4] differed from then state-of-theart computational results by three orders of magnitude; the discrepancy spurred improvements in both experiments and computational methods, so that there are now multiple methods for measuring the conductance properties of a single molecule, and the discrepancy between experiment and today's more physically realistic theoretical calculations is less than an order of magnitude.

The theory, modeling, and simulation portfolio being developed and applied in nanoscience ranges from first-principles electronic structure methods, through molecular dynamics with millions of atoms, all the way to finite element methods applicable at macroscopic sizes [5]. Corresponding to the range of length scales is a range of time scales from femtoseconds (electronic structure methods), through nanoseconds (atomistic simulations) and microseconds (mesoscale methods), to seconds (finite element methods) and beyond. While many of the needed theoretical and computational tools exist for systems of a specific size, a great challenge is to find accurate methods to bridge length and time scales, so that the accurate information obtained by electronic structure methods near defects or interfaces can be passed to more coarsegrained methods to model the properties of larger samples or composite materials. All of the present methods for specific length scales benefit from leading-edge computational facilities that permit the study of more realistic models of larger systems. However, there is also a need for new physical insights that can be translated into new, more efficient algorithms. Indeed, individual ingenuity often has had more impact than improvements in computer hardware, particularly at the formative stages in the development of a new scientific area.

There are numerous opportunities for computation and theory to impact nanoscience. Figure 3 shows an example in which the modeling of the synthesis requires complex transport, reaction, and growth mechanisms. The figure illustrates one of the beautiful morphologies of silicon oxide nanowire assemblies synthesized by chemical vapor deposition of silane with a molten gallium catalyst [6]. Although the nanowire assemblies formed in different temperature ranges have different morphologies, they are all composed of a spherical liquid-gallium ball and a silicon oxide nanowire bunch that grows out from the lower hemisphere surface of the gallium ball. To predict the structures produced in such a three-phase reactor is a formidable challenge for theory and simulation, yet it is a typical problem in nanoscience.

Another important challenge is self-assembly, where the goal is to design molecular building 


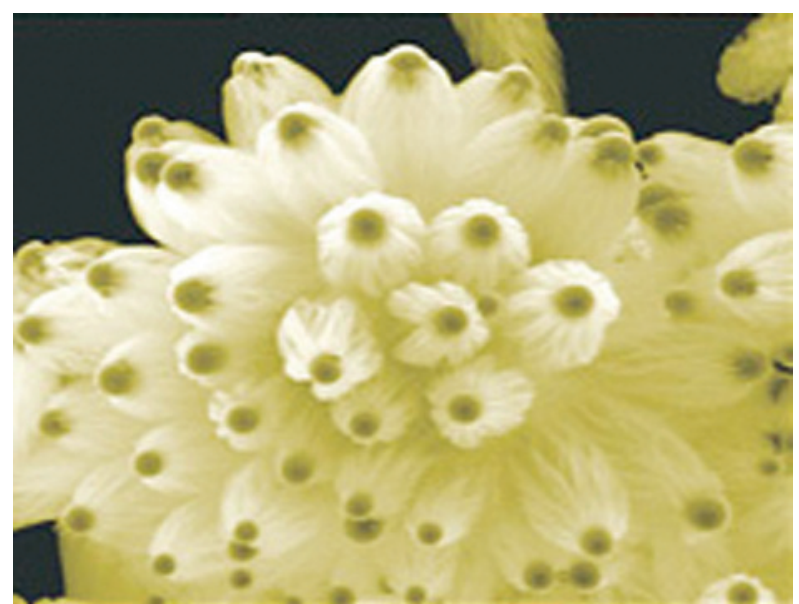

Figure 3. Example of the carrot-like morphology of $\mathrm{SiO}_{\mathrm{x}}$ nanowire assemblies, one of five different morphologies obtained in different temperature ranges during molten-gallium-catalyzed synthesis in a threephase reactor [6]. The gallium ball at the end of each structure is 4 to 5 microns in diameter. (Reprinted with permission from Z. Pan et al., "Temperature dependence of morphologies of aligned silicon oxide nanowire assemblies catalyzed by molten gallium," Nano Letters 3, 1279-1284. Copyright 2003 American Chemical Society.)

blocks that can undergo spontaneous organization into well-defined and stable macroscopic structures, typically held together by non-covalent bonds, including van der Waals interactions and ionic bonds, and often in the presence of water [7]. Natural biological systems are the supreme examples of self-assembly, and some recent approaches utilize biological molecules (such as DNA) as the scaffolds for directing self-assembly $[8,9]$. Examples of nanostructured materials produced in this manner include nanoporous catalysts, nanostructured block co-polymer systems, and DNA-assembled quantum dots. The theoretical and computational tools to describe self-assembly span the range from atomistic simulation to mesoscale and beyond. First-principles quantum electronic structure methods may be used to accurately determine the interatomic forces and this information can then be used to create forcefields needed for accurate molecular simulations of much larger systems.

Research on nanostructured materials for photonics is also on an explosive growth curve, fueled by the interplay between theory, computation, and experiment. The basic equations describing classical electromagnetic fields and the quantum mechanics of electrons are being harnessed to accurately design complex structures to control optical radiation over length scales comparable to the wavelength. For example, greatly enhanced local fields have been predicted in nanoscale tapered metallic plasmonic waveguides for use in ultrasensitive single-molecule spectroscopy on surfaces [10]. Also, scientists are discovering that photonic band gap crystals and "left-handed" metamaterials have remarkable properties that will lead to novel devices to control light in much the same way that semiconductors are designed to control electronic currents. The development of highly nonlinear nanoscale optical materials will open entirely new few-photon technologies for measurement, communication, storage, processing and display of information. Those technologies require comprehensive theoretical, computational, and experimental understanding of the basic science.

Many of the new frontiers and opportunities outlined in this report are closely related to nanoscience. Spintronics, single-molecule electronics, nanoporous catalysts, and biomimetic materials are tremendously exciting topics for Basic Energy Sciences. Indeed, nanoscience is pervasive and will impact nearly all areas of BES. The advancement will be greatly accelerated by the close partnership of theory, computation, and experiment; particularly in nanoscience, where models and simulations are often essential to unravel the complexity of the data acquired. In some cases theory and simulations may even lead the research. For example, the molecular dynamic simulations of Cao et al. of a carbonaceous material called a graphitic carbon inverse opal (Figure 4) predict that it may have a high capacity for hydrogen absorption [11]. The field of nanoscience is just beginning, and the future promises to be productive, competitive, and exciting.

\section{Correlated Electrons in Solids}

The collective behavior of electrons in solids leads to many remarkable properties and technological applications. Classic examples are superconductivity and magnetism. Yet, in many important cases, this collective behavior is not understood because of the great challenge of describing systems of strongly interacting electrons and ions.

High temperature cuprate superconductivity provides theorists with a number of puzzling 


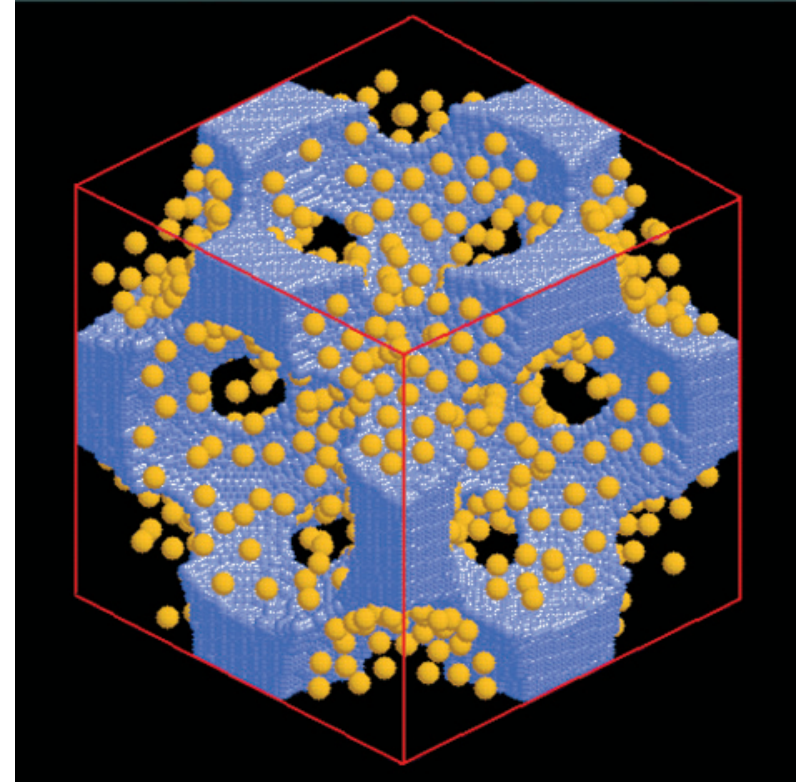

Figure 4. Simulation-based prediction of structure for a carbonaceous material that has a large capacity for adsorbing hydrogen. (Reprinted with permission from D. Cao, P. Feng, and J. Wu, "Molecular simulation of novel carbonaceous materials for hydrogen storage," Nano Letters 4, 1489-1492. Copyright 2004 American Chemical Society.)

phenomena. The cuprates at first glance seem to be rather simple systems. They are composed of metallic $\mathrm{CuO}$ planes separated by insulating spacers. A single partially filled energy band characterizes the $\mathrm{CuO}$ planes. Yet from this modest origin emerges very complex behavior (Figure 5), not only high-temperature superconductivity, but also antiferromagnetism, metal/insulator crossovers, charge inhomogeneity, and the pseudogap phase (a state of matter whose nature is not yet agreed upon).

Understanding the underlying physics of high $T_{\mathrm{c}}$ superconductors requires not only novel experiments, but also innovative theory and the development of new computational methods. For instance, we do not have an exact solution for the twodimensional Hubbard model (the simplest model used to describe the cuprates). We do not even know whether this model has a stable superconducting ground state. With further advances in the methodology of quantum Monte Carlo simulations, this important question may be answered. Another example of the complexity of the problem is the temperature dependence of the Fermi surface
(Figure 6). With a long-term perspective, the BES research portfolio could have the experimental, theoretical, and computational resources to collectively solve the "cuprate problem."

Magnetism is another important area where the understanding of correlated electronic states would have a profound effect. Magnetic materials are crucial for spintronics, for proposed quantum qubits, and for a host of many technological applications ranging from large permanent magnets for motors to nanoscale sensors, yet the theoretical and computational difficulties of dealing with strongly correlated electronic states have hindered progress in many magnetic materials. The basic problem is easily formulated, and simplified approaches such as the Hubbard model have led to considerable insight into the breakdown of single particle and mean field descriptions. Now, where standard DFT has proven inadequate, new computationally intensive theoretical approaches such as dynamical mean field theory and quantum Monte Carlo are leading to new insights and the promise of quantitative descriptions of real material properties. The development of these techniques is occurring just when

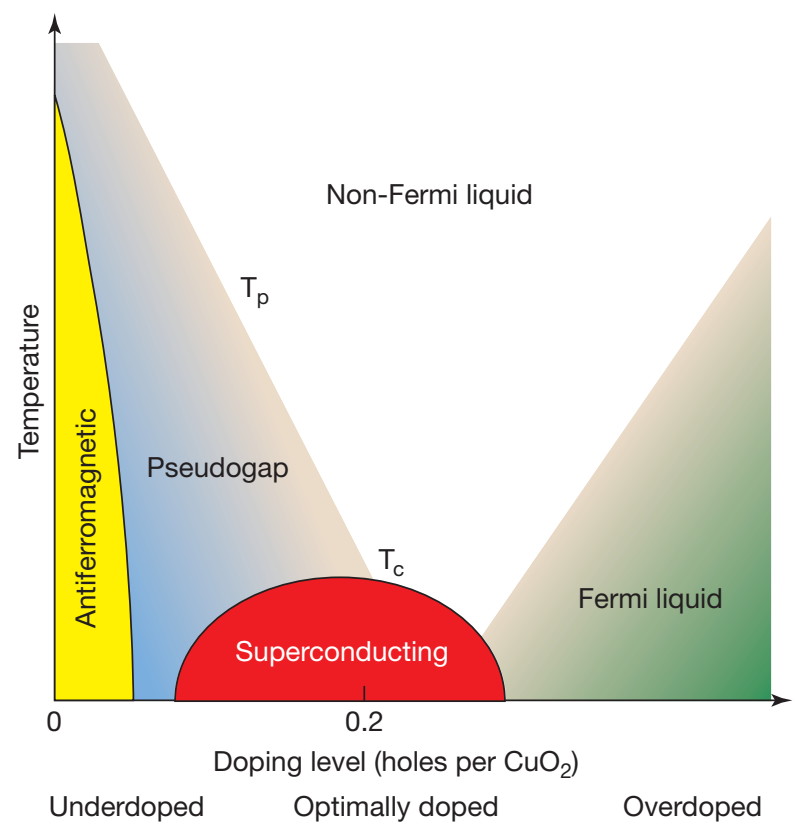

Figure 5. The phase diagram of a typical high-temperature superconductor, showing the parent, undoped antiferromagnetic ground state and how it evolves into several unusual and not yet understood phases as a function of temperature and doping. 


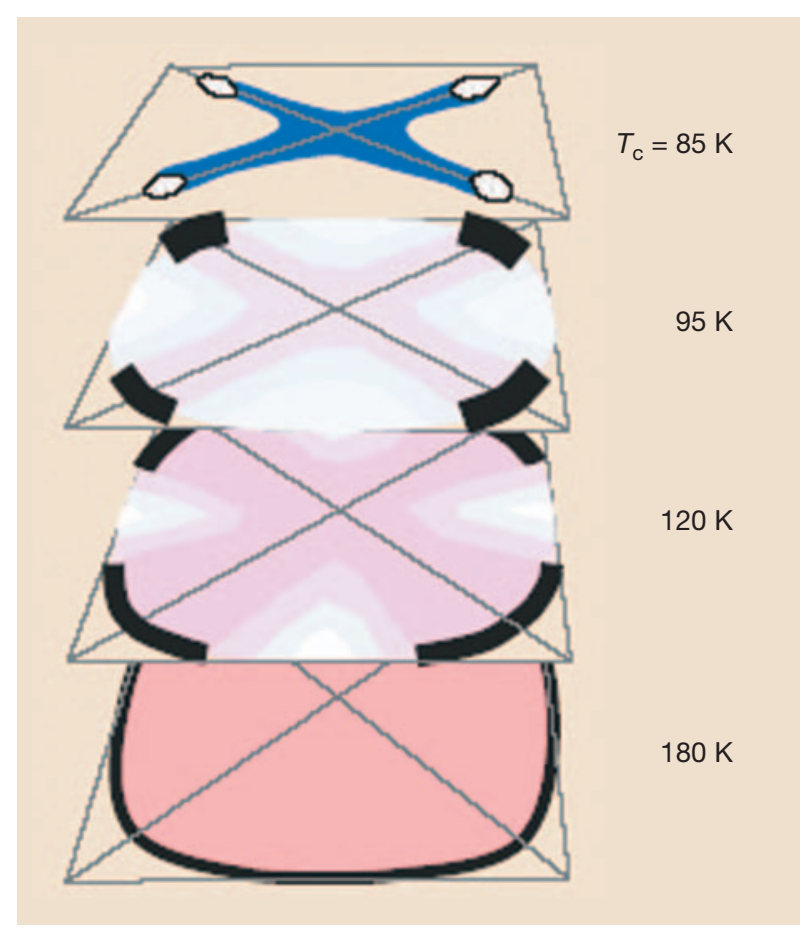

Figure 6. Destruction of the Fermi surface of a high-temperature cuprate superconductor as the temperature is lowered from $180 \mathrm{~K}$. Below the superconducting transition temperature of $85 \mathrm{~K}$, the surface breaks into isolated points, or nodes. There is currently no accepted theory to account for this behavior. (Adapted from a News and Views article by Piers Coleman, Nature 392 , 134 (1998).)

new neutron and magnetic x-ray scattering facilities are generating data on local moments and nontrivial excited states that require sophisticated analysis and accurate theoretical interpretation.

\section{Excited Electronic States}

The accurate description of molecular excited electronic states in both the gas and condensed phases is critical to a number of forefront research programs at DOE. Excited electronic states play key roles in photosynthesis; the production of new fuels, such as hydrogen, by photocatalytic means; and the efficient conversion of sunlight to electrical energy. Such states are formed when ionizing radiation interacts with aqueous systems, metals, and amorphous materials-systems of particular interest in modeling nuclear waste containment and in understanding the growth of defects in the walls of nuclear reactors. The molecular dynamics of a reacting system, such as the combustion of fossil fuels, are significantly affected by the presence of molecules in electronically excited states.

Calculations of the structure and dynamics of molecules in electronically excited states remain extremely challenging, particularly because both electronic structure and dynamics must frequently be treated quantum mechanically. Electronic structure methods are generally based on the BornOppenheimer (BO) approximation, which assumes an adiabatic separation of the motion of the heavy nuclei from the fast motion of the electrons. Calculation of the BO potential energy surfaces for excited states requires a high-level treatment of electron correlation. Breakdown of the BO approximation leads to dynamic coupling of different electronic states. A variety of processes, in which energy is transferred between electronic and nuclear degrees of freedom, require the evaluation of this coupling, and this capability is not yet a standard feature in many quantum chemistry codes.

Major advances in electronic structure methods have been made over the past decade, which, given access to the required high-end computing resources, enable calculations on increasingly large molecular systems. The importance of conical intersections, geometries where singularities occur in the nonadiabatic coupling operator, has emerged. Conical intersections form multidimensional seams, adding to the complexity of describing multistate dynamics [12]. A promising alternative to the $\mathrm{BO}$ approach for interfacing structure with dynamics is the direct calculation of diabatic states and their local, off-diagonal couplings (see Figure 7).

Advances in dynamical methods are required as well. Accurate quantum scattering approaches are limited to small numbers of atoms, and extensions to larger polyatomic systems necessitate progress in the development of approximate methods for treating nuclear dynamics. Direct dynamics methods, in which electronic energies, couplings, and gradients are computed on the fly, have been implemented for studying the dynamics of lowlying excited states. For higher excited states with multiple couplings, new approaches need to be developed.

Electronic excitations in condensed phase systems present an even more complex computa- 


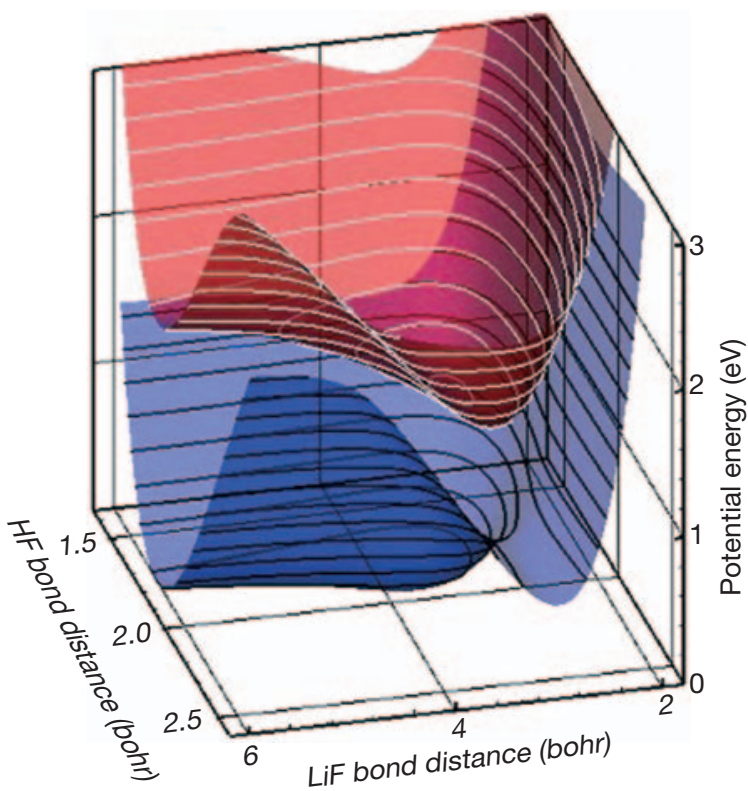

Figure 7. Non-Born-Oppenheimer dynamics on coupled LiFH potential energy surfaces. The ground state surface (blue) has a van der Waals well corresponding to $\mathrm{Li}$...FH, while the excited state surface (red) has a strongly bound excited state complex. The excited state complex can decay to form $\mathrm{LiF}+\mathrm{H}$ (front right of the plot) or nonreactively to form Li + HF (back left). (Image courtesy of A. Jasper and D. G. Truhlar.)

tional challenge than for gas phase systems. Such excitations can change the charge distribution in a molecule, which in turn perturbs the surrounding environment, such as a solvent. The solvent molecules, no longer in equilibrium, start to rearrange, profoundly affecting the subsequent dynamics of the excited molecule. Figure 8 illustrates the fact that solvation can have a large effect on excited state lifetimes by changing the characteristics of the relevant conical intersection. In solids the excitations can form localized defect states. In systems subjected to high dose rates of ionizing particles or energetic photons, the interaction of defects to form extended defects is important in understanding nuclear waste containment.

Treating electronically excited states in gasphase processes presents a significant computational challenge, one that can be addressed today using leadership-scale machines with emerging algorithms. Advances in computer architectures and the

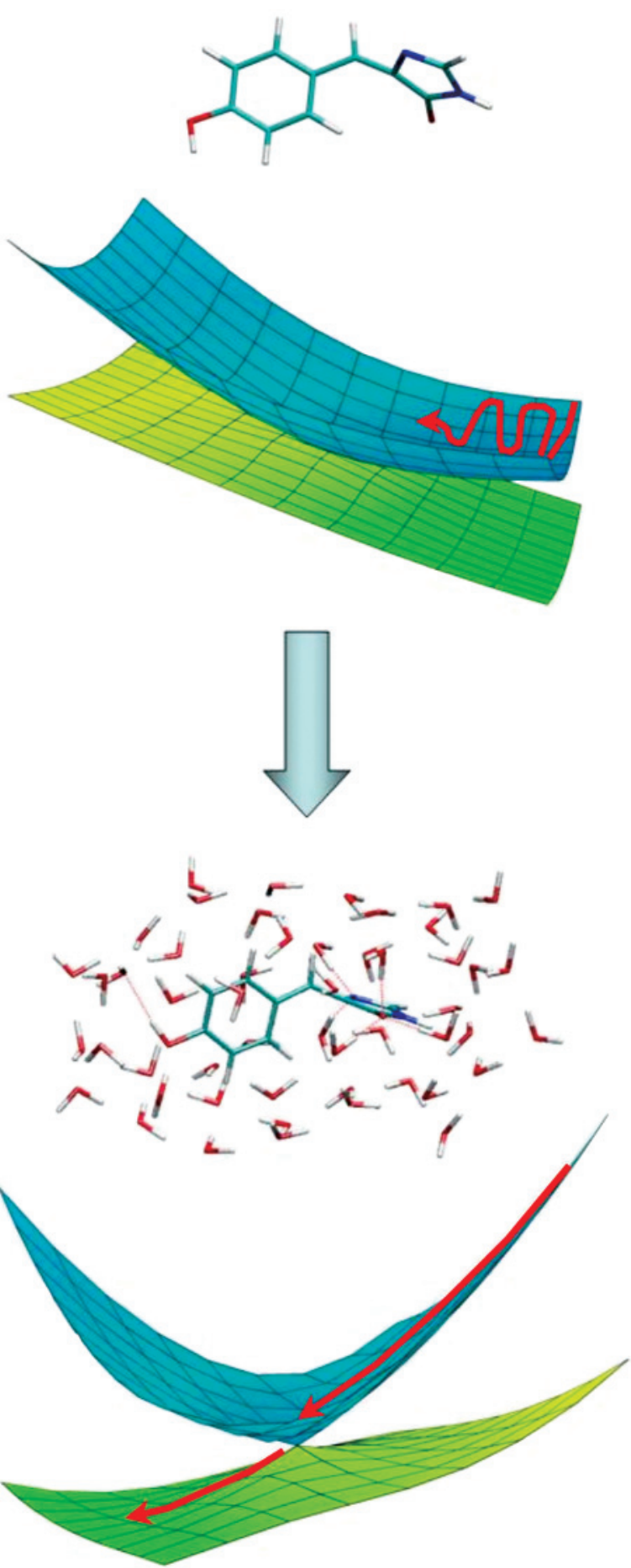

Figure 8. Quenching of the excited state of the chromophore of green fluorescent protein in isolation (top) and in a microsolvated environment (bottom). Solvation shifts the position of the conical intersection and makes the crossing more easily accessible. (Image courtesy of T. J. Martinez.) 
development of new methods will enable applications to larger, more complex molecules. Treating electronically excited states in condensed phase systems is a major theoretical and computational challenge and will require new approaches and computational tools as well as access to the high-end computing resources needed to validate the proposed methods.

\section{Defects in Solids}

Real materials have defects. Materials properties such as strength, transport, fatigue resistance, and magnetic hysteresis are greatly influenced by those defects. In general, these properties cannot be understood in terms of isolated "average defects," and it is necessary to understand the static and dynamical organization of microstructural defects and their collective behavior. This immediately raises issues of time and length scales. For example, plastic deformation of crystalline materials involves motion of large populations of line defects (dislocations). The dislocations interact with each other and with other defects in the material, especially grain boundaries, in a complicated and nonlinear fashion. One consequence observed is that yield strength exhibits a maximum as a function of the grain size. There is not yet a theoretical first-principles understanding of this phenomenon, which presumably arises from pinning of dislocation motion at boundaries between the crystallite grains. One reason for the lack of an adequate theory is the long-range but weak strain interactions between dislocations, which means that it is impractical to address the problem at the atomistic level. Another reason is the long-range topological character of some defects, which strongly affects their behavior. Therefore, it is essential to consider the problem at long length scales and seek insight from numerical simulations allied with novel statistical mechanics analysis.

Another example of the importance of collective behavior of defects, also drawn from the activities in the Computational Materials Science Network, is that of interfaces (see Figure 9). The organization of defects in solids is not static but changes with application of driving forces such as deformation, phase transformations, and elevated temperature. Impurities, in particular, affect the motion of interfaces at surprisingly low concentrations, often a few parts per million. Thus, although (a)

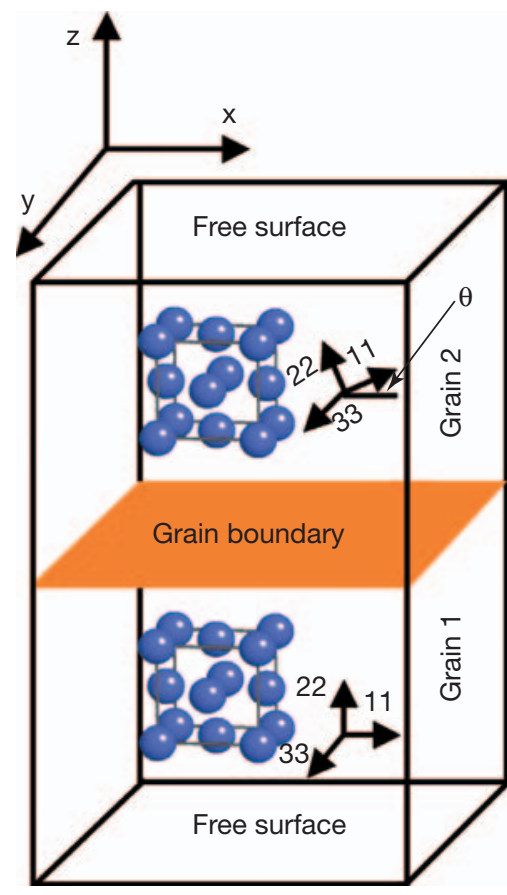

(b)

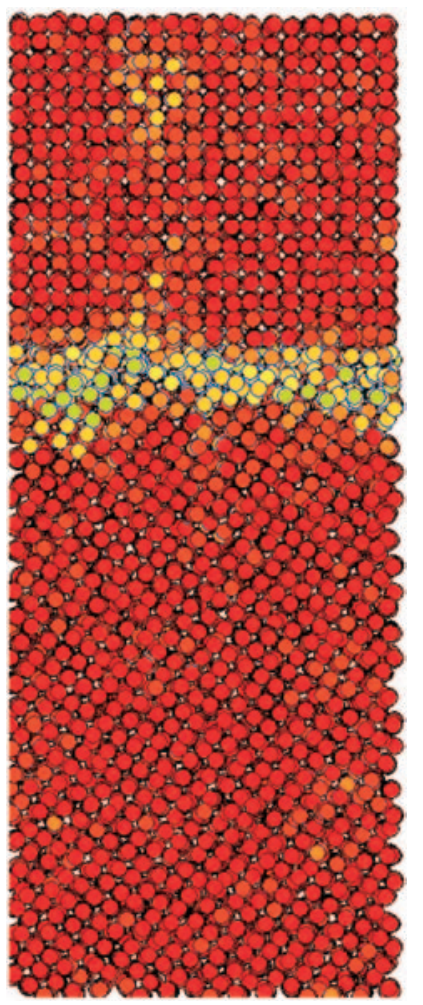

Figure 9. (a) Schematic showing the atomic orientations within two fcc crystallites (grains) whose interface forms a grain boundary. (b) A snapshot of the corresponding molecular dynamics simulation showing the 12-fold (fcc) coordinated atoms as red, and the atoms at the grain boundary with different colors, indicating lower coordination. (Image courtesy of David Srolovitz.) 
atomistic simulations are essential for ascertaining the local equilibrium and kinetic properties of interfaces, understanding the collective behavior requires theory and modeling at longer length and time scales. Forefront research now involves stateof-the-art atomistic calculations of interfacial properties (Figure 10) and coupling these results directly into mesoscale models of microstructural evolution. Such coupling of length scales is reinvigorating research in this area, for example in evaluating the differences between stored energy driving forces
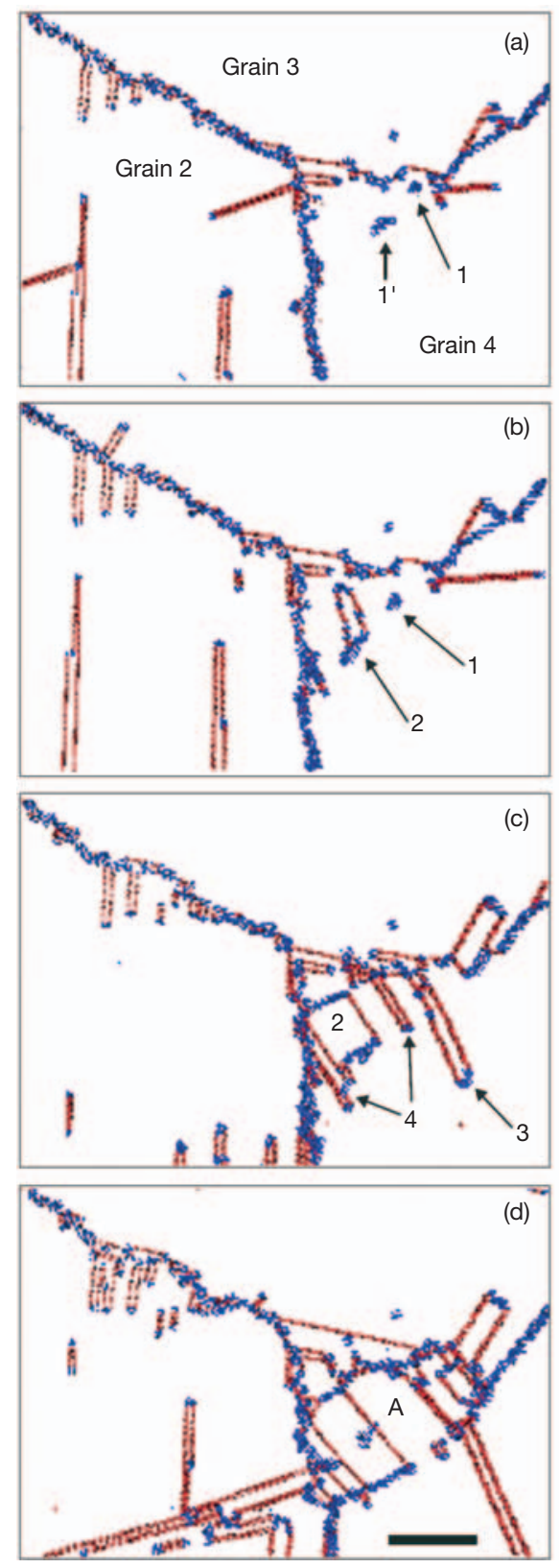

and curvature driving forces for boundary migration. At a more general level, the role of impurities that can strongly affect equilibrium and kinetic properties of materials is receiving new attention for control of solidification, recrystallization, precipitation, and transport properties.

The molecular dynamics and statistical mechanics methods which are the primary computational tools used for these studies scale exceptionally well with modern parallel computers. Simulations of complex microstructures with millions of atoms or more are leading to new insights and new theoretical models. There are now tremendous opportunities for discovering the scientific basis for some of the oldest materials science questions of how materials properties arise and how they can be controlled.

\section{B. OPPORTUNITIES AND CHALLENGES IN QUANTUM CONTROL}

Studies of fundamental interactions and understanding how to control or use them are vital building blocks for reaching the ultimate goal of controlling complex systems. Quantum control can take many forms, such as the manipulation of molecular orientation, the entanglement of quantum states, and the alignment of electron spins.

Figure 10. Results of a million-atom molecular dynamics simulation of aluminum, showing successive snapshots of the vicinity of the triple junction connecting grains 2,3 , and 4 , demonstrating the mechanism by which the new grain, $A$, is formed as strain on the "sample" is increased. Four distinct processes labeled 1-4 are revealed; these are described in V. Yamakov et al., "Dislocation processes in the deformation of nanocrystalline aluminium by molecular-dynamics simulation," Nature Materials 1, 45-49, 2002. Red dots denote atoms in an hcp environment. Blue dots denote atoms that are "defected," that is, neither in an fcc nor hcp environment, including atoms with broken nearest-neighbor bonds. Scale bar: $10 \mathrm{~nm}$. (Image courtesy of authors.) 
The ability to use lasers to trap and cool dilute atomic gases to ultracold temperatures has produced Bose-Einstein condensates (BECs) of a number of atomic species and has enabled the exploration of a variety of phenomena. Crafting electromagnetic pulses to break particular bonds or to align certain electrons to produce a desired outcome begs for theoretical input and guidance. Treating the dynamics of the electronic structure of atoms, molecules, and solids in strong electromagnetic fields is, of necessity, nonperturbative, and the accurate solution of the time-dependent Schrödinger equation demands tremendous computational resources. Opportunities for discovery in the BES portfolio which can be classified as "control" include control of energy, matter, and information at the quantum level; ultrafast physics and chemistry; and magnetic spin systems and single electron devices, all of which are discussed below.

\section{Control of Energy, Matter, and Information at the Quantum Level}

One of the most exciting and rapidly evolving areas in physics today is the field of ultracold quantum degenerate gases. The Physics Nobel Prize in 1997 recognized the innovative laser cooling and trapping techniques leading to the laboratory creation of dilute, ultracold gases of alkali atoms. The Physics Nobel Prize in 2001 recognized the first experimental formation and observation of BoseEinstein condensation in gases of rubidium and sodium. And the editors of Science magazine listed the formation of molecular condensates made up of fermionic atoms as one of the most significant breakthroughs of 2004 [13].

A topic that has been extensively studied is the behavior of Bose-Einstein condensates (BECs) and, more recently, degenerate Fermi gases at magnetic field strengths in the vicinity of a two-body Feshbach resonance. An intriguing element is the ability to use a controllable parameter in the Hamiltonian of the system /usually the magnetic field strength, $B$ ) to vary the particle-particle interactions, changing the system from a weak-coupling, perturbative regime into a strong-coupling, unitaritylimited regime. Such a system with a "knob" which can "dial up" a particular interaction strength offers an opportunity to deepen our understanding of the very interesting crossover problem in condensed matter physics, in which a system evolves from
Bardeen-Cooper-Schrieffer (BCS)-like pairing when atom-atom interactions are comparatively weak into a strong-coupling regime as the interactions are made strongly attractive. Eventually the system evolves into a BEC of molecules, making this BCSBEC crossover problem extremely rich (Figure 11). There is yet to emerge a single unified theoretical description that embraces both the weak- and strong-coupling limits. This is an area ripe for study, and it may hold the promise of elucidating a number of problems in many-body physics, including perhaps the mechanisms and detailed behavior of high- $T_{\mathrm{c}}$ superconducting materials.

The close interaction of theory with experiment in this area is extremely fruitful, driving scientific advances. For example, theorists first proposed the idea of the "atom laser" and shortly afterward several different laboratories had demonstrated this concept. In 2000, the theoretical work describing the idea of "stopping light" was followed several months later by the experimental demonstrations of the concept. Controllable optical lattices exhibit the periodicity or quasi-periodicity of many condensed

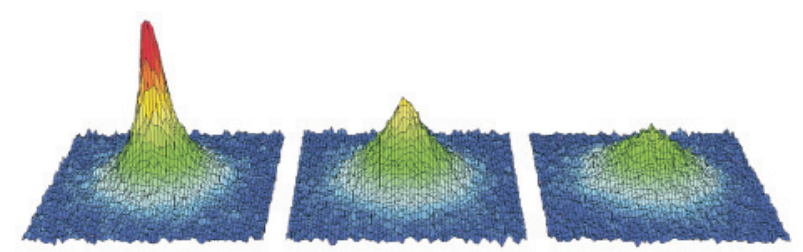

Figure 11. Experimentalists modify the interactions in a many-body system of fermionic atoms by varying an external magnetic field near a two-body Feshbach resonance. The far right figure shows an ultracold gas of 100,000 potassium atoms with weakly attractive interactions, predominantly a degenerate Fermi gas. In the middle figure, the magnetic field has been lowered, such that the stronger attraction has begun to turn the gas into a "fermionic condensate" of Cooper-like atom pairs. In the far left figure, the magnetic field is lowered farther and the fermionic condensate emerges even more clearly, driven by the stronger attraction that has been dialed in. (Reprinted with permission from C. A. Regal, M. Greiner, and D. S. Jin, "Observation of Resonance Condensation of Fermionic Atom Pairs," Phys. Rev. Lett. 92, 040403, 2004. Copyright 2004 by the American Physical Society.) 
matter systems and appear to give a promising realization and testbed for an important class of theoretical methods used widely in many-body physics.

The quantum control of a reactive scattering or photochemical process by the application of specifically tailored electromagnetic pulses is an exciting possibility, with seemingly unlimited practical applications. A corollary of this theme is the reverse, namely the use of molecular properties to modify and control light pulse properties, such as temporal structure and phase chirp. To date, some examples of control of chemical reactions have been demonstrated. Particularly promising for experimentalists has been the use of genetic algorithms to utilize in real time "self-learning" methods that steer a reaction in a desired direction. It has been difficult, however, to extract an underlying theoretical understanding from such algorithms. Another area is the identification of molecular systems with particularly simple and stable temporal behavior, such as the increasingly studied rotational revivals, which can be exploited to cause an interaction with a laser beam at particular moments when the molecule is highly aligned. This has already been shown to permit a new class of experiments on aligned molecules, and it also can be used to modify the nature of a probe light pulse that passes through an aligned molecular gas.

The use of the quantum mechanical notion of entanglement to enhance the ability to process and transfer information is at the heart of the rapidly growing areas of "quantum information/computation" and "quantum communication." Scientists have been intrigued by the possibility of using the superposition of quantum states to achieve advances in certain kinds of computation and to improve current encryption schemes.

In the field of quantum information and computation, conceptual theory is leading the way. Theorists were the first to suggest the idea of creating entanglement in an ion trap, and experimentalists were able to demonstrate this capability. In subsequent work, other theorists developed a method to implement quantum "gates" and entangle quantum states of ions using a bichromatic laser pulse. The experimental demonstration of this idea was successful, resulting in the entanglement of four ions. In liquid state NMR, the theory was worked out for pulses to control gates, and seven entangled nuclei within a molecule were used in a remarkable implementation of Shor's quantum factorization algorithm.

Thus the proof of principle for quantum computing has been demonstrated in the laboratory at the simplest level, but scaling up to large enough systems to exploit quantum entanglement for serious applications requires a great deal more understanding of how to control decoherence in complex quantum systems. The drive for such understanding may lead to exciting, serendipitous applications in other areas. It is an outstanding opportunity to deepen our understanding of decoherence, dissipation, and entanglement.

The physical and chemical tools to study these problems and assess the viability of the "quantum information/computation" enterprise lie within the Basic Energy Sciences research portfolio. This is an area which is currently attracting the attention of some of the best scientists in the field, along with their students. It is cross-cutting research, with efforts already ongoing in leading groups in atomic physics, condensed matter physics, quantum optics, and chemistry.

\section{Ultrafast Physics and Chemistry}

The vision that drives ultrafast science-probing, following, and ultimately controlling the movement of atoms and electrons-seems closer to being realized now than ever before. The potential benefits of realizing this vision include the ability to image biological molecules, steer chemical reactions, manipulate the dynamics of carriers in semiconductors, and develop new x-ray laser schemes. The successes of ultrafast science over the next decade, whether in the well-established field of femtochemistry or in the new area of attosecond pulses, will depend increasingly on an intense interplay between experiment and theory, often aided by large-scale computation. New investments in theory for ultrafast physics and chemistry offer the opportunity to continue the rapid progress of the past decade and to optimize the much larger investments in ultrafast experiments both in the laboratory and at the national facilities.

Ultrafast science is propelled by dramatic advances in light source technology. Femtosecond lasers operating at infrared frequencies can now produce amplified pulses that approach the fundamental limit of one optical cycle in duration, about 3 fs. Using the highly nonlinear interaction of such 
a laser with an atomic gas, experimentalists have been able to produce attosecond (sub-femtosecond) pulses at much shorter XUV wavelengths, both as single pulses and coherent trains of subpulses. Subpicosecond sources in the x-ray region are under development at national facilities. In the U.S., prominent examples are the Sub-Picosecond Photon Source (SPPS) and the Linac Coherent Light Source (LCLS) at the Stanford Linear Accelerator Center. These sources allow for the deposition of very large amounts of energy in atoms, molecules, and clusters on extremely short time scales. Understanding the effects of this rapid energy deposition, and how to use it to probe atomic and electronic motion, is the challenge for theory.

The advent of these ultrafast light sources, in the laboratory and at national facilities, has stimulated theorists and experimentalists to explore exciting new applications. In recent work researchers have been investigating the possibility of using attosecond electron wave packets, generated by the high-intensity, ultra-short laser-atom interaction to probe nuclear processes [14]. Another concept involves harnessing the coherent oscillation of an ion resulting from dissociative photoionization of a muonic molecule to stimulate nuclear reactions in adjacent nuclei, thus creating the opportunity to monitor and control nuclear decay [15]. Still another idea is to use high-harmonic generation to resolve the motion of electrons in a molecule by mapping out the spatial distribution of the highest occupied molecular orbital [16]. This involves techniques to monitor the scattering of an ionized electron wave packet off the outer orbits of the electrons in a molecule as the molecule rotates.

Over the past decade scientists have used broadband infrared lasers to coherently control a select few reactions in small molecules. This has firmly established the principle that femtosecond pulses with appropriate amplitude and phase distributions can be used to excite tailored quantum wave packets of nuclear motion. This application of "coherent control" to steer molecular dynamics and chemical reactions along specific reaction pathways has been articulated in the preceding section (Control of Energy, Matter, and Information at the Quantum Level). While there exist formal theories for determining optimal control pulses, the actual calculation of such pulses for interesting chemical reactions is a daunting task, involving many of the challenges articulated in other sections: the difficulty of calculating electronically excited states, the importance of conical intersections in determining the molecular dynamics, and the complexity of the calculations requiring high-end computational resources for determining simultaneously the electronic structure and the nuclear dynamics.

In the science of attosecond pulses, opportunities for theory fall broadly into two categories: the generation and optimization of attosecond sources, and the new science made possible by attosecond sources. Theoretical study of attosecond pulse generation requires the simultaneous solution of the time-dependent Schrödinger equation (TDSE) and the Maxwell wave equation (MWE) in the time domain. This is a very large-scale computational problem, but one that is well adapted to large cluster architectures. However, the parameter space to be studied is huge, and although the bandwidth exists to make pulses as short as 10 attoseconds, utilizing that bandwidth will require substantial advances in our understanding of the full generation process.

Even more challenging than attosecond pulse generation is the study of the time evolution of quantum systems excited, ionized, or probed with attosecond light. Because of their short time scale, these pulses are ideally suited to studying the fast interactions between electrons, thus permitting the study of the excitation and ionization dynamics of correlated systems (Figure 12). Phenomena such as autoionization and shakeup ionization can be addressed in the time domain. With appropriate pulse shaping, coherent control of electron motion may be possible. Calculating optimal scenarios for electron control will be a key part of the design and interpretation of experiments with such pulses.

The LCLS will take ultrafast science to a truly new regime by delivering millijoule pulses of $\sim 8$ $\mathrm{keV}$ photons in 200 fs bunches of extremely short sub-femtosecond pulses. Two aspects of proposed LCLS experiments offer great challenges for theory. The first is the prospect that LCLS will have sufficient photon flux and time resolution to do ultrafast, single-molecule x-ray diffraction experiments. Theory and computation will be essential for the interpretation of single-molecule diffraction patterns, as x-rays are scattered by the electrons, not by the nuclei. Thus the x-ray diffraction image will be time-dependent. The intense radiation creates 


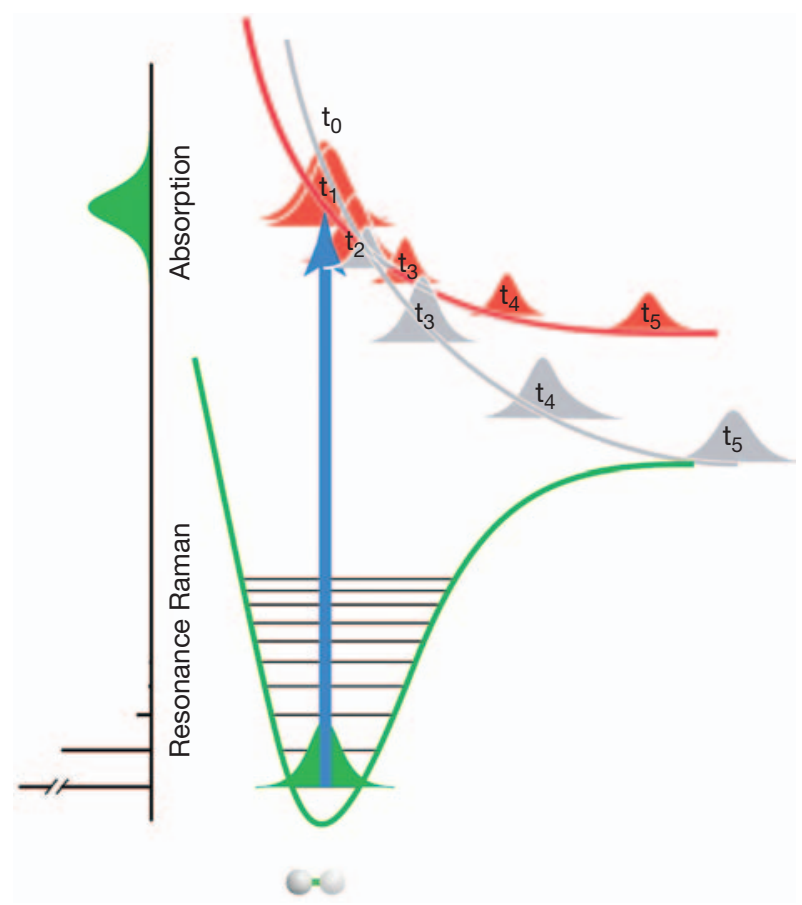

Figure 12. Schematic representation of a photo-dissociation process that includes potential energy surface hopping. The initial wave packet is prepared on the optically active red excited state, which is crossed by the dark gray state. As the wave packet approaches the crossing point, a fraction of its amplitude crosses to the gray state, changing its electronic character in the process. (Image courtesy of Robin Santra.)

inner-shell holes, followed by Auger processes, radiative decay, and collisional ionization by photoelectrons, all of which imply a time-varying electronic target during the $\mathrm{x}$-ray pulse. Theoretical input will be indispensable in reconstructing the molecular geometry that existed prior to the pulse.

The second challenge for theory is to calculate the effects of a focused LCLS beam on a target atom, molecule or cluster. The x-ray pulse will create multiple inner shell holes, leading to exotic excited states, which may be useful, for instance, in $\mathrm{x}$-ray laser schemes. The opportunity to study multiphoton ionization of inner shell electrons is new. No theoretical studies of this process exist for strongly bound, highly correlated inner shell electrons. Understanding the electron and photon emission from such experiments will require solving a strong-field, relativistic many-body problem, necessitating the development of new codes and access to forefront computing resources. Such an effort is needed even to understand the simplest atomic experiments which will be used as diagnostics for the LCLS.

\section{Magnetic Spin Systems and Single- Electron Devices}

Molecular electronic junctions are structures in which single molecules or small groups of molecules conduct electrical current between two electrodes [17]. If they could be the building blocks of novel electronic devices, this would open new frontiers to miniaturization. Combine this idea with the endless possibilities of synthesis and functionalization offered by chemistry, and it is easy to see that molecular devices may revolutionize the future of the electronics industry, which is running up against tremendous barriers in trying to keep conventional developments on pace with Moore's Law.

In addition to charge, electrons also carry a spin, and the possibility of manipulating the spins and controlling their flow opens exciting perspectives for new information storage and transmission devices linking information to spin orientation [18]. Since spins interact weakly with the environment via magnetic fields, spin excitations have a much longer lifetime than charge excitations. This property makes spintronic devices potentially attractive for applications such as quantum computers, whose practical realization depends crucially on long quantum coherence times. Spintronic devices rely on spin-polarized electronic transport, an effect that is already in use in existing devices made by ferromagnetic-nonmagnetic thin film layer junctions. The electric resistance of these junctions is strongly magnetic field dependent resulting in giant magnetoresistance, a new physical effect discovered in 1988, and subsequently exploited in modern hard drives for computers.

In spite of its enormous potential, nano-electronics is today mostly a hot topic for fundamental research and has not yet impacted technology in a dramatic way. One reason is lack of fundamental knowledge: our understanding of quantum transport in interacting many-electron systems is still in its infancy. Another reason is that experiments requiring accurate control of matter at the nanoscale are difficult. As a consequence we are still far from being able to mass-produce molecular devices with selected specifications. 
Experiments on molecular and nanoscale junctions measure the I-V characteristics, i.e., the current as a function of the applied voltage. A basic transport mechanism at the nanoscale is quantum tunneling, which can be either elastic or inelastic. Several fundamental questions on how tunneling occurs under given experimental conditions are still open. Major unresolved questions involve understanding how contacts and/or interfaces between different sections of a device operate, and how nanostructures, such as individual molecules, couple to macroscopic electrodes under non-equilibrium conditions. Understanding these phenomena is a very challenging task for theory because transport is a dynamic process, and even under steady state conditions it is a non-equilibrium phenomenon. Density functional methodologies are well established for ground-state and equilibrium phenomena, but it is not clear if they can be extended to non-equilibrium transport phenomena. Furthermore, some of the new phenomena that experiments have revealed in nanoscale devices, like the Coulomb blockade and the Kondo effect, require treatments that go beyond the simple effective single particle approximations that are currently used in equilibrium electronic structure calculations (Figure 13).

In some circumstances a description limited to the electronic degrees of freedom is inadequate, and one needs to understand how tunneling electrons couple to local vibrational modes. This is crucial, for instance, to understand inelastic electron tunneling spectroscopy of molecules adsorbed at surfaces [19]. Selective excitation of local vibrational modes, due to tunneling electrons under applied bias, may lead to control of the chemical reactivity of adsorbed molecular species, which is one of the goals of quantum control research. Finally, understanding how quantum systems couple to macroscopic reservoirs is a general issue in all transport phenomena. In some cases the difficulty of dealing with a quantum mechanical system coupled to a bath may be bypassed if the bath does not require explicit treatment; however, there do exist situations that require an explicit treatment of dissipation and coherence-breaking phenomena. This is an area where progress is needed even at the level of the basic mathematical equations.
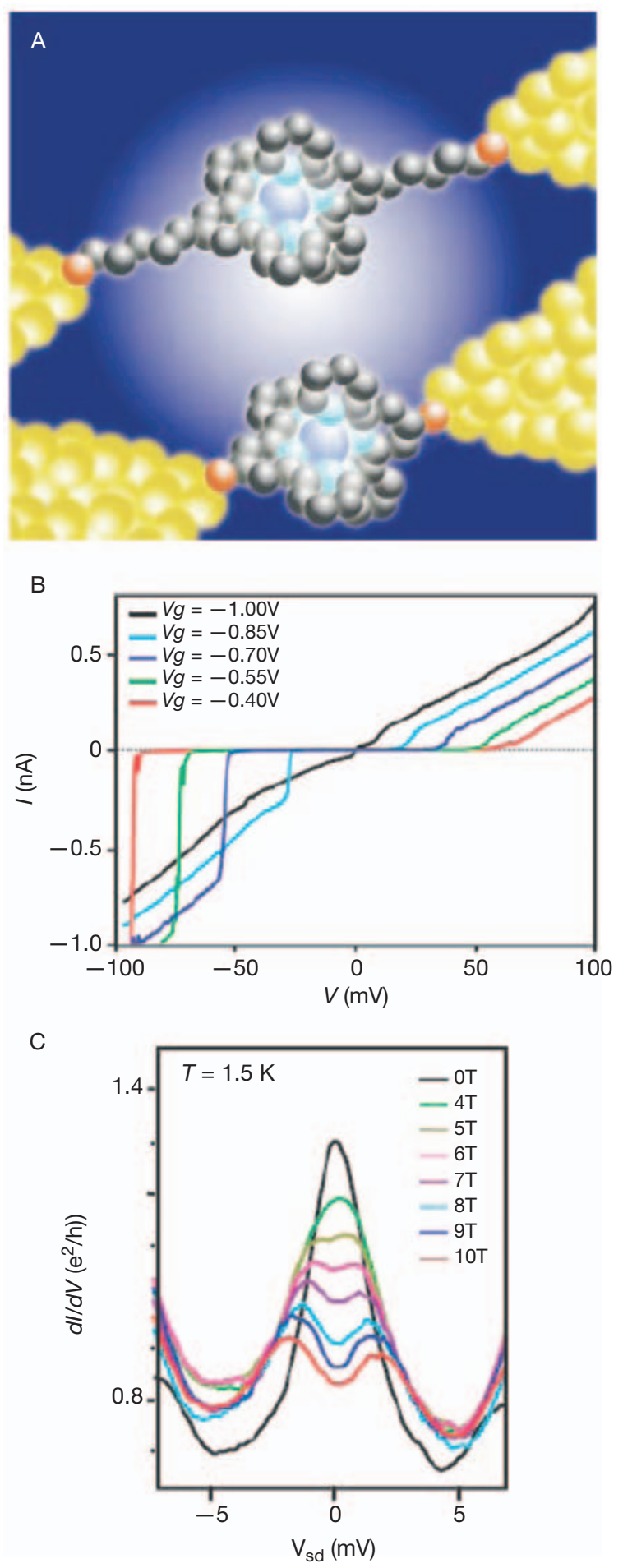

Figure 13. (A) Schematic of a single molecule junction between two electrodes. The two lower graphs report transport measurements using similar structures. (B) The current-voltage characteristic showing the "Coulomb blockade." (C) The effect of magnetic fields on the Kondo resonance. (After J. Park et al., "Coulomb blockade and the Kondo effect in singleatom transistors," Nature 417, 722 (2002).) 


\section{OPPORTUNITIES AND CHALLENGES IN CONTROL OF COMPLEX SYSTEMS}

The following topics involve both complexity and control in exploring and understanding their various aspects: biomimetic materials and energy processes, and control of chemical transformations.

\section{Biomimetic Materials and Energy Processes}

For sustaining life on earth, nature has produced an astonishing variety of biomolecular architectures, refined by more than three billion years of natural selection. Biomimetics, the science of mimicking natural processes in form or function, holds great potential for the Basic Energy Sciences. Macromolecular assemblies have evolved to perform a myriad of functions in living organisms, including efficient conversion of light energy into chemical energy; chemical sensing and signal transduction; catalysis of solution-phase chemical reactions under ambient conditions; generation of mechanical force from chemical energy in molecular motors; and construction of light, strong, and wear-resistant structural materials.

Biomimetic energy processes provide promising routes to a sustainable, clean energy supply, including artificial photosynthesis for producing electrical energy from sunlight. The determination of three-dimensional structures of bacterial photosynthetic components, along with ultrafast spectroscopic measurements of electronic couplings and energy transfer rates, has enabled the development and calibration of quantum mechanical descriptions of light harvesting and energy transfer in photosynthetic organisms (Figure 14). These, in turn, have inspired new advances in theory that provided a more accurate description of energy transfer. A challenge now is the extension of theoretical and simulation approaches to implement emerging concepts in the design of artificial photosynthetic processes. An important example would be hydrogen production by biological and biomimetic processes with sunlight as the driving force and water as the electron source. Theory and computation are needed for protein structure prediction and for refinement algorithms leading to structurefunction relationships of hydrogenases, while largescale electronic structure calculations and molecu- lar dynamics simulations are needed to elucidate the mechanisms of light capture, hydrogen production, and inactivation by oxygen poisoning in the molecular machinery.

Molecular motors are proteins that use chemical energy to generate mechanical forces that are used by living organisms to carry out a variety of physiological processes. The fundamental question of how elementary, localized chemical reaction events generate directed mechanical force in a regime where viscous forces far exceed inertial forces remains unanswered. This is an extremely active field with tremendous opportunities, pushed on the experimental side by single-molecule experiments employing laser tweezers and fluorescence and atomic-force microscopies, and pushed on the theoretical side by new developments in the theory of stochastic processes, nonequilibrium statistical mechanics, and simulations of the chemical reactions and their mechanical consequences on molecular and mesoscopic scales.

The ability to understand and control biomolecule-surface interactions is essential for potential applications such as the fabrication of sensors, mechanical actuators, and molecular electronic components. While there have been pioneering investigations using classical force fields for simulations of specific protein-surface interactions at the atomic level (e.g., Figure 15), many fundamental challenges remain concerning the nature and consequences of biomolecule-surface interactions that could be addressed by theory and modeling. For example, electronic structure calculations can be used to develop better empirical force fields; Monte Carlo simulations and long time-scale molecular dynamics simulations with coarse grained models can be used to predict whether a biomolecule will undergo significant conformational changes and possibly be inactivated when interacting with a surface; and atomistic simulations could be used to predict the orientation and accessibility of reactive regions of biomolecules on specific surfaces. A recent study illustrated the potential power of such simulations, showing the interaction of a single molecule of DNA moving through a pore in an inorganic membrane (Figure 16).

Nature offers numerous examples of materials with high strength-to-weight ratios. Biocomposite materials are an important class consisting of nanocrystalline inorganics in organic (protein) 

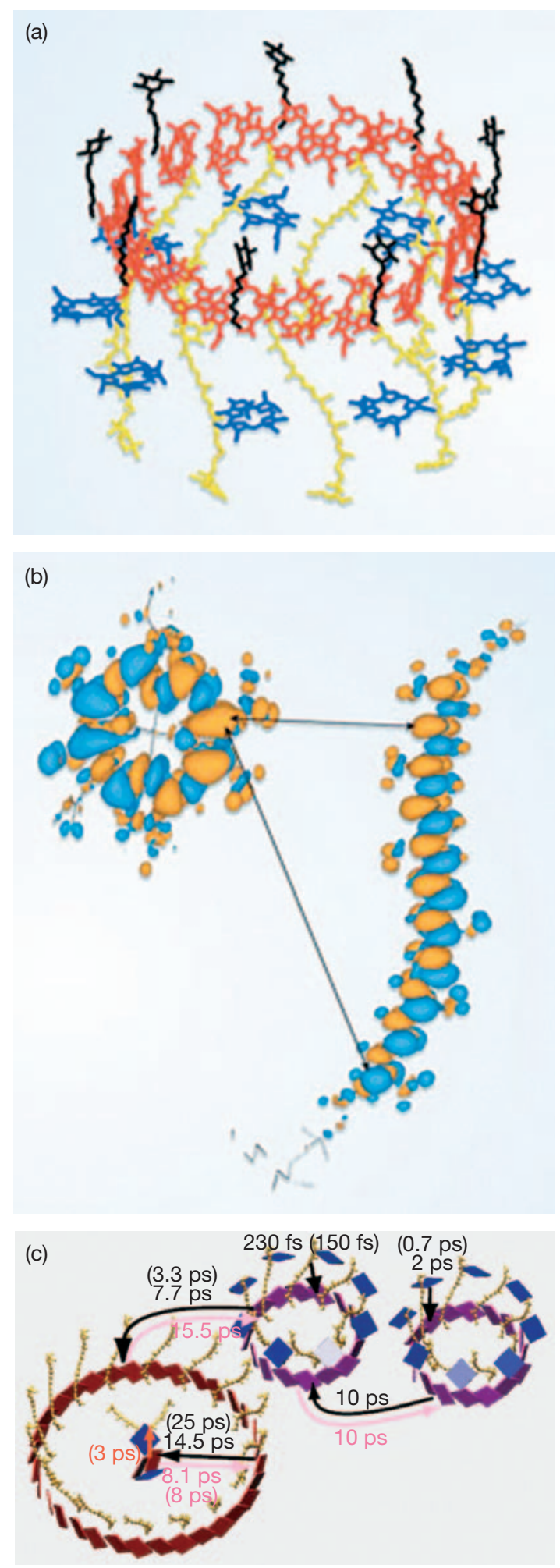

Figure 14. (a) Chromophores in a model of light-harvesting complex (LH2) from the bacterium Rhodopseudomonas acidophila. (b) Electronic interactions as seen in the transition densities of LH2 bacteriochlorophyll (left) and carotenoid (right) molecules calculated from ground- and excited-state wavefunctions. (Reprinted with permission from G. R. Fleming and G. D. Scholes, Nature 431, 256-257. Copyright 2004 Nature Publishing Group.) (c) Times for excitation transfer between the various pigments in the photosynthetic unit. All times were determined through quantum-mechanical calculations on the basis of the atomic level structures. Experimentally measured times are shown for comparison in parentheses. (Reprinted with permission from T. Ritz et al., Proc. Idea-Finding Symposium, Frankfurt Institute for Advanced Studies, pp. 177-188. Copyright 2003 EP Systema, Debrecen.)

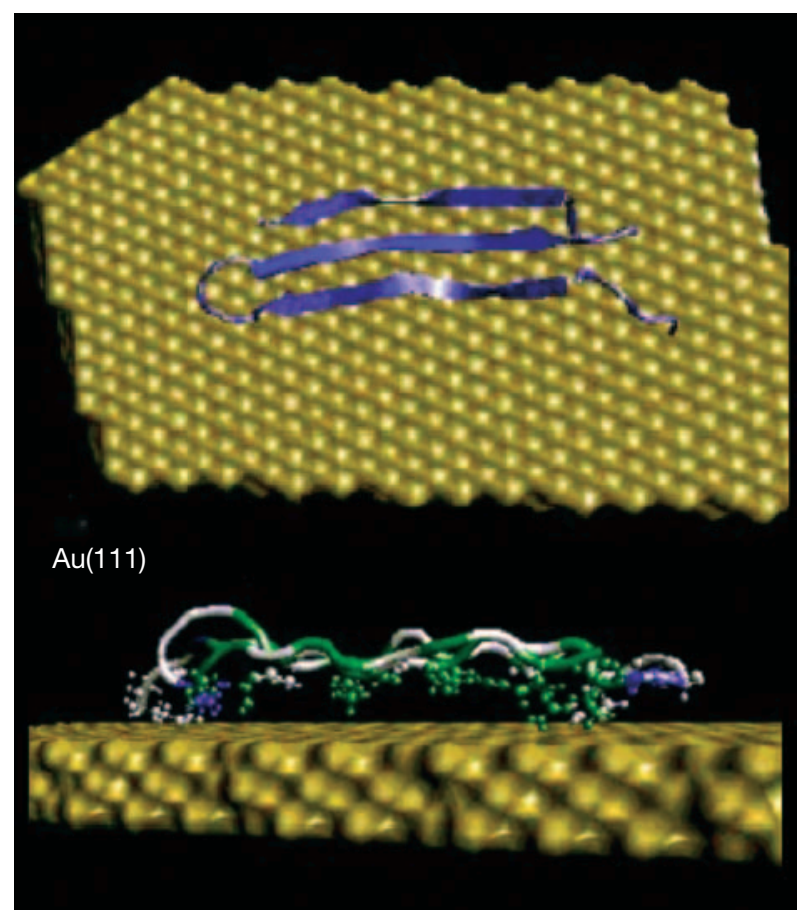

Figure 15. Molecular model obtained by structure prediction and molecular dynamics simulation of a designed gold-binding protein interacting with a gold (111) surface. Simulations suggested that the binding of the peptide to the $\mathrm{Au}(112)$ surface is less effective due to the penetration of solvent between the peptide and the surface. (Reprinted with permission from $\mathrm{M}$. Sarikaya et al., Nature Materials 2, 577-585. Copyright 2003 Nature Publishing Group.) 


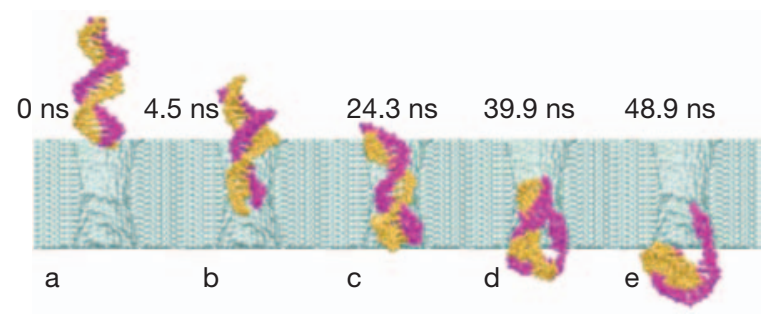

Figure 16. A team of electrical engineers and computational biologists has demonstrated the feasibility of using a nanometer-diameter pore in an inorganic membrane as a transducer for the detection of single molecules of DNA. When an electric field is applied across the membrane, a DNA molecule enters the pore, blocks the current, and eventually translocates across the membrane. The relationship between DNA translocation and blocking current has been established through molecular dynamics simulations. (Reprinted with permission from A. Aksimentiev et al., Biophys. J. 87, 2086-2097. Copyright 2004 Biophysical Society.)

matrices. Examples include sea shells (based on calcium carbonate), diatom skeletons (based on silicates), and bones (based on calcium phosphate). An understanding of how the hierarchical structures and interactions in these materials translate into their remarkable physical properties could lead to strategies for producing tailored structures with characteristics that far exceed those of existing man-made materials. As in the examples above, there is need for theory and computation to develop accurate understanding of the bonding and reactions at the atomic level, and through coarse graining or other schemes, to understand the relationship between structure, dynamics, and the mechanical properties of these systems.

Computational modeling of biomolecules has evolved dramatically in power and scope due to advances in theory that have led to new simulation algorithms and analysis protocols, and advances in hardware that have enabled larger systems to be treated more accurately and on longer time scales. In this field, highly optimized, user-friendly simulation packages (e.g., CHARMM, AMBER, NAMD, GROMACS), suitable for describing a wide variety of biomolecules with classical force fields, are widely distributed to a growing community of both theorists and experimentalists. Many of these programs have been developed outside of BES, but their availability and use has helped create col- leagues in the biological community who speak the language of atomistic and coarse-grain modeling, and who are invaluable as members of interdisciplinary teams tackling outstanding problems relevant to biomimetic materials and energy processes.

\section{Control of Chemical Transformations}

The control of chemical transformations, encompassing the entire panoply of chemical reactions and rearrangements in the gas phase, in liquids, in solids, and on surfaces, is an age-old dream, originally pursued by alchemists. Modern-day experimental techniques have explored a rich variety of chemical processes, challenging theorists to formulate quantitative methods which have accurate predictive capabilities. The sum of steady progress in the development of theoretical and computational tools over the past decade is offering exciting new possibilities for describing chemical transformations at the molecular level. An essential requirement for progress in this area, however, is access to both leadership-class and mid-scale capacity computing, coupled with algorithm development and an infrastructure that would support the integration and maintenance of large codes.

Chemical dynamics, which is the study of chemical transformations, is at the heart of many areas of interest to DOE, including combustion; photosynthesis; catalysis for efficient energy production; contaminant degradation in the environment by natural and remedial processes; reactions that determine the fate of byproducts of energyproducing and energy-utilizing processes; corrosion in nuclear reactors promoted by reactive radical species, such as $\mathrm{OH}$; release of hydrogen, for use as fuel, from its chemically bound forms; degradation of materials used for nuclear waste entombment; and destruction of hazardous waste by supercritical oxidation.

Significant progress has been made in developing computational tools for understanding and predicting the reaction mechanisms and rate constants of gas-phase reactions. Four areas of research may be identified as critical to the study of chemical dynamics: electronic structure, to obtain the atomic forces and potential energy surfaces; statisticaldynamical theories such as transition state theory and variational transition state theory, for calculating the overall rates of reaction; quantum mechani- 
cal scattering theory for calculating state-to-state processes; and master equations, for inferring overall rate coefficients and their pressure dependences from the above. Despite the progress that has been made in these areas over the past two decades, some key issues are not fully understood. An example is a lack of understanding of the factors controlling soot production in combustion processes. Although this was once considered too complex for modeling, we have now reached the point where a molecular-level understanding may be achievable. A second critical issue is extrapolation of rate constants measured in one temperature regime to another regime of more interest for a particular application.

Researchers are beginning to perform the first predictive, direct simulations that reveal the fundamental turbulence-chemistry interactions in realistic complex flames using detailed representations of the chemistry and transport. With continued improvements in the chemistry and transport models, new algorithms and enhanced computing power, long-standing problems in combustion science, such as predicting pollutant emissions and quantifying pressure effects on flame dynamics, can be understood.

An even larger number of scientific opportunities are associated with developing computational tools required to understand and control chemical reactions in condensed-phase environments and at interfaces. These are the environments in which chemical catalysis occurs. The challenges of understanding interfaces include predicting the reaction mechanisms and kinetics of nanoscale and interfacial processes, and theoretically describing heterogeneous electron transfer processes. Other challenges in condensed phases involve quantifying the effect of equilibrium and nonequilibrium solvation on reaction rates, and gaining a fundamental understanding of radiation effects on condensed matter.

Notable advances have been made in our ability to treat reaction dynamics in complex systems (Figure 17). These advances include both the development of new methods and computational software packages for electronic structure, dynamics, and kinetics modeling, primarily for gas-phase reactions. Important progress has also been made in integrating different methods (e.g., electronic structure with dynamics) to allow for more efficient modeling of complex reactions and mechanisms. A major opportunity exists to develop shared codes that further integrate these methods and make these programs available to the scientific community. Further challenges for computational chemical dynamics include:

- Extending theoretical approaches for analyzing high-resolution and single-molecule spectroscopies used to probe the molecular-level mechanism of energy-producing and energyutilizing processes.

- Improving the quantitative reliability of electronic structure algorithms (wave function theories, density functional theory, and hybrid methods) for treating ground and electronically excited states.

- Developing and extending new electronic structure methods that allow information from the atomic level to be transferred to more complex systems, such as liquids,

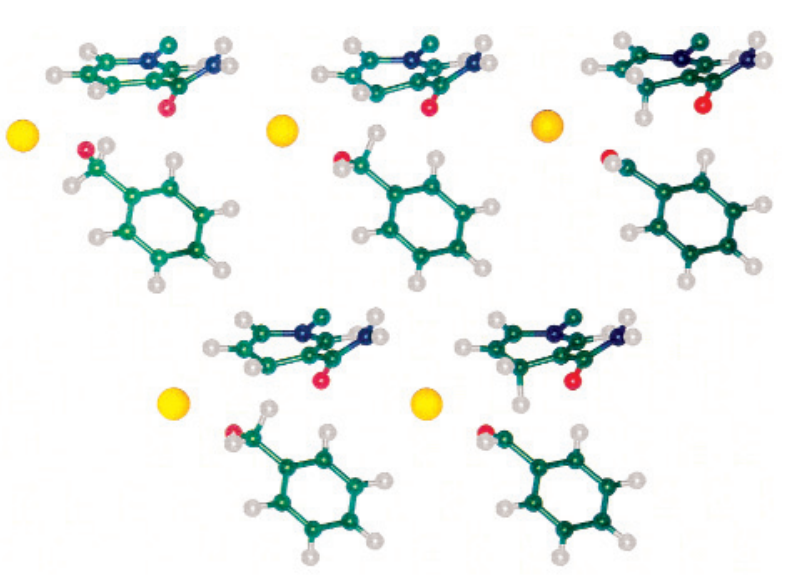

Figure 17. Variational transition state theory including quantum effects was used to describe the nature of the tunneling process in a complex hydrogen transfer reaction catalyzed by liver alcohol dehydrogenase. The top row shows reactant, transition state, and product, while the bottom row shows the starting and ending termini of the dominant tunneling path, with the time sequence of the reaction following the "W" of yellow dots. The potential energy surface is calculated by treating 54 atoms by quantum mechanical electronic structure methods, and 5,506 protein, coenzyme, and solvent atoms by molecular force fields. (Reprinted with permission from C. Alhambra et al., "Quantum dynamics of hydride transfer in enzyme catalysis," J. Am. Chem. Soc. 122, 8197-8203. Copyright 2000 American Chemical Society.) 
amorphous solids, complex fluids, nanostructured materials, and interfacial systems.

- Inventing new methods that bridge length scales in seamless ways to couple classical mechanical inhomogeneous macroscopic responses to the quantum mechanical electronic structure of molecular and nanoscale systems.

- Developing efficient ways to sample the dynamics of systems over time scales much longer than molecular scale times, including the coupling of classical and quantum dynamics.

- Advancing our understanding of intermolecular interactions and how to model them efficiently, accurately, and realistically in large ensembles from nanoscale systems to bulk materials.

- Discovering strategies to deal with the "inverse problem," where the composition and structure of a new material are designed to express a desired property. 


\section{Connecting Theory with Experiment in BES: Accelerating Discoveries and Furthering Understanding}

A key part of the scientific mission of the Department of Energy is the construction and operation of large experimental facilities for the scientific community. We have identified a need for stronger coupling of theory and computation to the experiments under way at these BES facilities as a major theme, expressed by both theorists and experimentalists in the community. Theory and computation could perform several roles in partnership with experiment at these facilities. In areas where the experimental techniques and theoretical understanding are mature, theory and computation play the critical function of interpreting experiment, making it possible to perform ever more advanced investigations. In areas where the experimental methods are both novel and still rapidly evolving, theory plays the role of leading experiment, suggesting new areas of inquiry and proposing new kinds of experiments.

At each of the five nanoscience facilities presently under construction, we find an effort to build an accompanying theoretical and computational capability as part of the development of the facility. We applaud the fact that the leaders of these facilities have recognized that theoretical and computational research is critical to the success of the nanoscience facilities. In public testimony we heard that in many cases, results and interpretation of nanoscience experiments are impossible without theoretical input and analysis. Without associated theory programs, the nanoscience facilities would be at risk of not realizing their full potential. These facilities are facing a combinatorial explosion of the degrees of freedom for synthesis. That is, there are so many possibilities to structure, pattern, and grow nanoparticles, thin films, or biomimetic materials, that some design guidance from theory and simulation is a tremendous advantage. With five separate nanoscience centers, there is need for coordination among the different theory and computation components so that their efforts can be leveraged.

In contrast to the nanoscience centers, we find that at the existing light and neutron sources there appears to be little conscious or systematic effort by BES to stimulate and support, with targeted resources, theoretical partnerships with experimental efforts. We believe that this situation puts DOE's ability to extract the maximum scientific benefit from those facilities at risk.

The nascent theory programs at the Spallation Neutron Source are not yet formulated sufficiently for the Subcommittee to form recommendations. This facility, the most expensive of all the experimental facilities of the Office of Science, is focused on an array of scientific problems across chemistry and materials science, and for many of these, mature theoretical and computational approaches already exist. The Office of Science thus has much to gain by partnering new theory and computational programs with SNS experimental efforts, both to interpret the results of planned experiments and to spark the design of new experiments.

We have found compelling evidence of the need for theory programs associated with the fourth-generation light source under development at SLAC, the DOE Linac Coherent Light Source. For this facility, theory and computation must at this stage play the role of leading and driving experiment. In addition, there are important opportunities for theoretical studies to inform and perhaps dictate the designs and specifications of the experiments themselves. Section IV.B.2, "Ultrafast Physics and Chemistry," describes the exciting science and helps to clarify the opportunities and need for theoretical efforts that can identify and propose new 
physical processes that will become accessible using ultrafast and ultra-intense light sources.

The Subcommittee discussed several ways in which the facilities might increase interactions among experiment, theory, and computation. Inhouse theory groups are common at major European facilities (e.g., the European Synchrotron Radiation Facility in Grenoble), and have the advantage that experimentalists using the facility need only walk down the hall to engage a knowledgeable theorist in discussions. The foremost example of this model in the United States was Bell Laboratories prior to the 1980s. Another mode discussed for more directed and perhaps shorter-term projects involves funding university researchers. Sponsoring workshops and extended visits for theorists can also be effective. With the terabytes of data being generated or expected for many modern experiments, a serious in-house computational facility is essential, and the proximity of computer scientists with high-capacity computers is a major attraction for many theorists. Because there is no single model for more closely coupling theoretical and computational efforts to experiments at these facilities, and because the task is beyond the capabilities of the Subcommittee as it is constituted, we will not make recommendations specific to each facility.

A major new thrust is needed in BES to significantly augment the theoretical and computational programs coupled to experimental research at these facilities. It is clear that such efforts should be incorporated in the plans for new facilities from the outset. At the facilities under construction, BES should energetically turn its attention to ensuring that by the time they are operating, such theoretical and computational programs will be in place. At the existing facilities, increased investments are needed to build theory efforts that strongly couple to the science that those facilities enable. All of these efforts should have the support of the respective user groups of each of these facilities.

Therefore, to be successful in more strongly connecting theory and computation with the experimental efforts at the facilities, BES will have to undertake an appropriately inclusive and vigorous planning process.

We observe in this context that large facilities impact benchtop science and vice versa. Thus large synchrotron x-ray sources have resulted in an increased demand for laboratory x-ray sources. Tabletop experiments in this area have, in turn, been the precursors to the best facilities-based experiments. A successful new effort that couples the BES programs in theory and computation to its experimental programs must also address the coupling to small-scale and single-PI experimental components of those programs. Only by creating a vision for a comprehensive investment in this area can BES expect broad support from both the experimental and theoretical communities. 


\section{The Resources Essential for Success in the BES Theory and Computing Enterprise}

\section{A. THE FULL SPECTRUM OF COMPUTATIONAL RESOURCES}

It has been recognized for well over a decade that a hierarchy of hardware resources is necessary to support computationally intensive research in all scientific disciplines. Scientific progress depends on ready access to all parts of that hierarchy and on the technological evolution of its components. This notion was expressed powerfully in a seminal report in 1993 to the National Science Board entitled "From Desktop to Teraflop: Exploiting the Lead in High Performance Computing," popularly known as the "Branscomb report" [20]. The components of the now-famous computing pyramid from that report have changed, but the concept endures.

An updated version of that pyramid of resources is shown in Figure 18. The first level of computing in this hierarchy is provided by desktop workstations that are dedicated to individual researchers to provide for data analysis, graphics, visualization, and small- to medium-sized jobs. At the next level today is cluster computing, which,

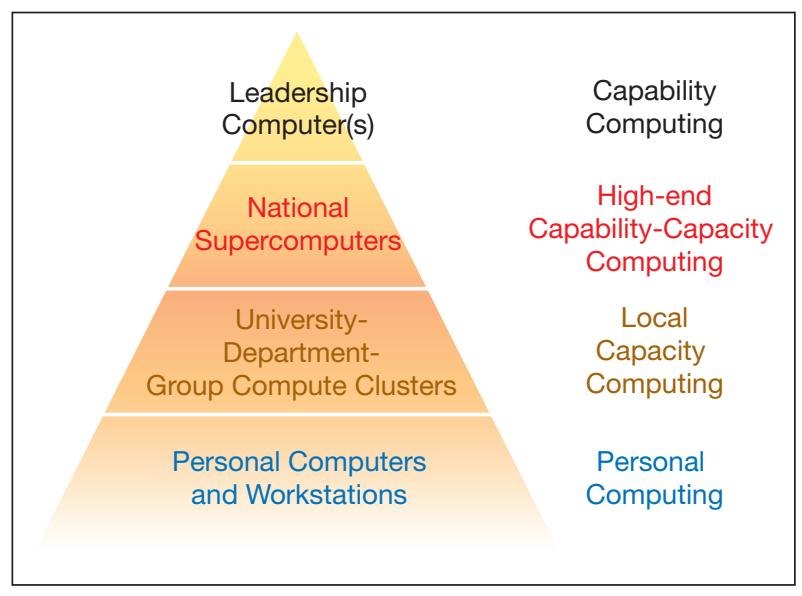

Figure 18. Updated version of the "Branscomb pyramid" [20] of computing resources. depending on the size of the cluster, can be the resource of a single research group, department, or university. The next level is particularly critical now in the age of widespread multi-teraflop computing, and is provided by national supercomputer centers. In the lexicon in which we distinguish "capability" computing that can only be done on large-scale facilities from "capacity" computing that must be done routinely, national supercomputer centers provide "capability-capacity" computing. We cannot perform teraflop-scale calculations on smaller resources, and a large segment of the BES community must do them routinely. Finally, at the peak of the pyramid today are "leadership-scale" computers. These are the truly unique resources that can enable a new class of computational science in the BES portfolio.

Cluster computing and midrange servers are playing an increasingly important role in the scientific enterprise. These resources allow for more efficient use of large-scale computing facilities, in addition to allowing for a significant amount of "production" computing. For example, smaller preliminary calculations performed on midrange computer systems can help identify the critical calculations that require access to large-scale computing resources. It is not unusual for the architecture of the midrange computers to be different than that of large-scale computing systems (e.g., symmetric multiprocessors [SMP] versus massively parallel processing [MPP]). These midrange architectures can frequently offer advantages for running calculations that require fewer computing cycles, but at the same time require access to large amounts of memory and disk space.

At this moment, the scientific progress of the BES theory community is being hindered by a lack of adequate computational resources at levels of this hierarchy from the midrange or cluster scale to leadership-scale computing. A number of algo- 
rithms that are common in the BES theory portfolio (for example, classical molecular dynamics, quantum Monte Carlo methods, classical Monte Carlo simulations, and density functional theory) are known to scale well to the largest numbers of processors on current supercomputers. Moreover, computer codes are in existence for all of these and other types of calculations that already run on these facilities. Reliable and plentiful access to both leadership-scale and terascale capacity facilities is required to enable this part of the current BES theory portfolio to achieve its full potential.

The path to petaflop computing capabilities is being pioneered in this country and in particular at the DOE national computing facilities. While the exact architecture or architectures that will emerge with those capabilities is not yet certain, we do know that they will involve tens of thousands of processors, and that their forerunners to be developed in the coming few years will involve thousands of processors. The ability to make use of those machines will be essential to many parts of the augmented BES theory and computation portfolio that this report recommends. A significant and growing part of that portfolio is characterized by algorithms that are known to scale well to these levels. These BES applications have the potential to make major and highly visible scientific advances in the near future if the appropriate resources and encouragement are given to this community. The BES portfolio offers a major and currently underexploited opportunity to the Office of Science in this respect.

Commitments for computer time and access at the high end of the computational hierarchy, however, need to be made available to theorists for several years at a time, so that the investment of time in rewriting and optimizing extensive computer codes for a particular kind of machine can yield significant scientific dividends. Those large commitments of computer time and support will be essential for the collaborative efforts that we expect to be necessary in some fields to make use of parallel computing with thousands of processors.

Collaborative efforts involving physical scientists, mathematicians, and computer scientists have been initiated in the Scientific Discovery through Advanced Computing (SciDAC) programs in BES, and their promise is described in the 2002 BESAC report entitled "Theory and Modeling in Nano- science" [5]. Such collaborative programs were also endorsed by the Advanced Scientific Computing Advisory Committee (ASCAC) in its report "HighPerformance Computational Needs and Capabilities in the Office of Science" [21], in which one of the recommendations reads: "ASCAC strongly supports coordinated Office of Science programs that tightly couple science, high-end computing, and applied mathematics." The Computational Materials Science Network (CMSN) in the BES Materials Sciences program is also a mechanism to bring together teams of scientists from different disciplines to solve important problems, often requiring high-end computational resources.

It should be noted also that there are single-PI efforts and small collaborations that are also able to make use of the highest end of the computing hierarchy. The award in the INCITE program of FY2004 to a BES researcher for quantum Monte Carlo studies is an example of such a single-PI capability. If they are to produce the scientific advances of which they are capable, these researchers and small collaborations in the BES portfolio will also require sustained access to the high-end facilities, including leadership-scale capabilities.

While some research in the BES portfolio is already poised to take advantage of computing on a leadership scale, other research groups may more profitably use cluster computing or smaller-scale centralized facilities. Their needs can be met by investments in local cluster computing and by capacity computing at the terascale. Capacity computing at the terascale (currently defined roughly as 1 to 5 teraflops peak power) is generally performed on one of the national computing facilities, and BES researchers are already major users of these facilities. In order to satisfy their needs, more such access will be needed. The issues of the reliability of those time allocations over a period of several years must be addressed, so that these BES researchers can make optimum decisions about their computing strategies.

\section{B. SCIENTIFIC CODES AS SHARED INSTRUMENTS}

Many of the computer codes that make it possible to express and exploit theory in all of the scientific areas in the BES portfolio can be viewed as large- 
scale instruments in their own right. In particular, to exploit high-end parallel computing, individual research groups or consortia must develop complex computer programs that frequently consist of tens or hundreds of thousands of lines of code. But with almost no exceptions, BES does not fund the maintenance, documentation, and evolution of those codes for the use of groups other than the one in which they were developed. These are demanding tasks that scientists cannot afford to undertake for the rest of their community without significant personal sacrifice of their own scientific research.

Nonetheless, scientific progress is being hindered by the lack of this class of shared software. Research groups in the Basic Energy Sciences frequently reinvent or redevelop methods and algorithms that may already exist in another research group. In addition, potentially important software tools developed by theorists are rarely exploited by experimentalists.

The process of developing large-scale scientific applications software is an iterative one, as the diagram in Figure 19 suggests. The theory community views the development of algorithms and software exactly as the experimental community views the development of experimental methods and instruments. Scientific application codes can evolve over a long period of time, during which their utility to a larger community can be established if they are documented and made available to other researchers. They can play the role of shared scientific instruments. Just as is the case with shared experimental instruments, they are most useful when other researchers can build upon them. Thus

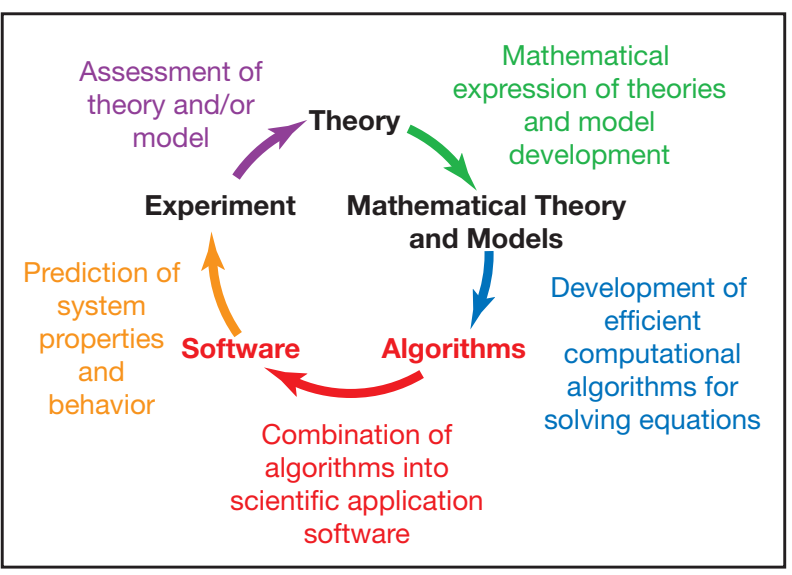

Figure 19. The iterative process of developing largescale scientific applications software. the sharing of such codes at the source level, where they can be modified and used as the building blocks of still larger codes, is essential. The Department of Energy has a long history of supporting the development of shared libraries, such as those that now form the heart of modern applications of numerical linear algebra (EISPACK, LINPACK, LAPACK, and ScaLAPACK). The model we suggest for more extensive shared scientific applications software is similarly noncommercial and open.

The Subcommittee received testimony regarding how this model is being used in other disciplines and supported by other Federal agencies. For example, the National Institutes of Health gives grants to individual PIs to provide "research resources" to the rest of their communities, and this mechanism is now being used to fund the maintenance, documentation, and distribution of molecular dynamics software for the computational biology community. The Department of Energy has an opportunity to establish a signature role in its support of science in the BES portfolio by encouraging and directly supporting the development of shared scientific applications software as a new kind of research facility.

The Subcommittee recommends that BES support the development and maintenance of scientific codes in the disciplines in its portfolio, just as it now funds the development of shared beamlines at its experimental facilities. This type of project would complement the projects now supported in theory and computation by BES, and would create community scientific software that would be shared and further developed by theoreticians and frequently used directly by the associated experimental communities. This style of support would distinguish BES support of the physical sciences from that of the other federal agencies, and it would be consistent with the mission of the Office of Science to create new scientific capabilities for the nation. In order to enhance the ability of BES theorists to take advantage of high-end computer architectures, there should also be opportunities for partnerships with applied mathematicians and computer scientists in the development of algorithms and software. Such activity would be consistent with the philosophy of the SciDAC program, a concept that appears to have considerable support in the community. 


\section{THE HUMAN RESOURCES: TRAINING FUTURE GENERATIONS OF THEORISTS}

Students and postdoctoral fellows are the lifeblood of Basic Energy Sciences. Advancing the boundaries of knowledge and creating innovative solutions to scientific problems, both now and in the future, depend on attracting and supporting the brightest students. The education of these students takes place largely in single-PI research groups at leading colleges and universities. Funding for students and postdocs is of primary importance in the single-PI and small, multi-investigator research groups, ensuring continuity in the research enterprise and the training of new generations of scientists.

In addition, research groups at colleges and universities need access to the appropriate computational resources. State-of-the-art codes developed by large consortia and running on leadership-class facilities should be made available to the university-based research groups, so that students and postdocs can become proficient in research involving high-end computation. Adequate support in the research being carried out in such groups is essential to the future well-being of the field and should be part of any overall strategy to increase support for theory and computation in BES. 


\section{Findings and Recommendations}

Theory, computation, and modeling have advanced to such a degree, and the new experimental programs and facilities are attacking problems of such complexity, that we cannot imagine any program or facility being successful without a sustained accompanying investment in theory from the outset. The role of theory is both to provide understanding and, increasingly, to lead experimental efforts. Theory and computation are now being used with greater frequency in the design and control of experiments, and we expect that trend to continue.

The findings of the BESAC Subcommittee on Theory and Computation are presented here in the context of our identification in this report of the growing importance of theory and computation to the goals of the scientific mission of the DOE.

FINDING: New opportunities for theory and computation have been created by the recent confluence of striking scientific successes and the appearance of specific new scientific frontiers. We have identified nine areas of opportunity spanning the range of the BES portfolio that have emerged strongly from the Subcommittee's investigations and the testimony it has received, although they do not exhaust the list of exciting new prospects:

- Nanoscience: providing early insight and guidance, and ultimately quantitative understanding, for the emerging new science and technology of materials designed, fabricated, and operating at nanometer length scales. Fundamental new theory is needed in many areas of nanoscience, but in other areas scalable codes implementing state-of-the-art theory, ready to take advantage of leadershipclass computers, are available today for application to outstanding and time-critical problems.

- Biomimetic materials and energy processes: synthesizing new bio-inspired materials and understanding photochemical and enzymatic processes leading ultimately to engineering-level control of biomimetic energy production.

- Correlated electrons in solids: developing theories and new computational methods that will contribute to quantitative understanding of spectacular phenomena such as high $T_{C}$ superconductivity, colossal magnetoresistance and exotic magnetic phases.

- Excited electronic states: improving and developing new theoretical and computational approaches to understand the structure and dynamics of electronically excited states in molecules, liquids, and solids and at interfaces, leading to better control of catalysis and radiation-induced processes.

- Control of energy, matter, and information at the quantum level: directing the outcome of chemically reactive processes with shaped laser pulses, tuning the interaction strengths of few-atom or many-body systems using external fields in quantum gases, and exploring the issues surrounding quantum entanglement and decoherence in quantum information science.

- Ultrafast physics and chemistry: optimizing new sources of attosecond light pulses and femtosecond electron pulses, and addressing the new physics accessed by those sources holds the promise of revolutionizing the study and control of highly excited electron dynamics.

- Defects in solids: developing theoretical and computational methods for bridging the atomic and mesoscopic length scales to understand and control important physical properties of materials such as strength, transport, fatigue, and magnetic hysteresis.

- Magnetic spin systems and single-electron devices: understanding the quantum- 
level processes that govern spin and electron transport in new multilayer materials and ultra-small devices being investigated for the next paradigm in computer technology.

- Control of chemical transformations: developing new theoretical and computational capabilities to quantitatively predict chemical reactions and rearrangements in condensed phases and complex environments that underpin new technologies in combustion, photosynthesis, catalysis for efficient energy production, and the chemistry of environmental remediation.

RECOMMENDATION: In all of these areas of opportunity, progress would be greatly accelerated by advances in fundamental theory to develop new paradigms to solve those problems, and BES should invest in such research. In many of these areas in particular, quantum control and information, ultrafast science, spintronics, and electronically excited states - new fundamental, conceptual theory is essential to progress.

RECOMMENDATION: A number of these areas are poised to exploit high-end computation, especially nanoscience, chemical transformations, electronically excited states, defects in solids, and correlated electrons in solids. Investments should be made to ensure the expansion of such computationally intensive research. Computation in these areas is essential to establishing and maintaining a leadership role for BES in both theoretical and experimental facets of these disciplines.

FINDING: We do not know all the equations, nor do we have all the mathematics and physical insights we need, and therefore we have not yet invented all the algorithms we need to solve the research problems in the BES portfolio. No computational facility that can be envisioned can solve these problems without new concepts and corresponding algorithms.

RECOMMENDATION: Enhancements in computation should be accompanied by enhancements in the rest of the theoretical enterprise. There is a basic unity of fundamental theory, modeling, and computation. Conceptual theory and computation are not separate enterprises.
FINDING: The recent trend to increase the coupling of theoretical and experimental activities in certain BES programs, for example at the Nanoscale Science Research Centers, is both proper and timely.

FINDING: However, at the existing light and neutron sources, there appears to be little conscious or systematic effort by BES to stimulate and support, with targeted resources, theoretical partnerships with experimental efforts. We believe that this situation puts at risk DOE's ability to extract the full scientific benefits from those facilities.

FINDING: At the facilities currently under construction (the Nanoscale Science Research Centers and the Spallation Neutron Source), some efforts have been made to incorporate theory into the planning for the facilities. However, these efforts are incomplete and uneven in scope.

RECOMMENDATION: We recommend that BES undertake a major new thrust to significantly augment its theoretical and computational programs coupled to experimental research at its major facilities. (A) When new experimental facilities (e.g., LCLS) are proposed by BES, the associated theory and computational efforts should be incorporated from the outset. (B) At its existing facilities, BES, with support of user groups, should upgrade theory and computation efforts affiliated with experimental programs. Such efforts could include personnel, resources, and support for a shared software infrastructure. (C) Increased investments in the BES programs are needed to build theory efforts that strongly couple to science at existing facilities.

FINDING: Much of the need for increased support for theory derives from qualitatively new experimental capabilities developed by individual investigators or small groups of investigators, and involving "tabletop" scale research. The increased complexity of systems that can be addressed with such capabilities has produced an unprecedented necessity for the intimate involvement of theorists in stateof-the-art laboratory experimental programs.

RECOMMENDATION: BES should enhance its support of theoretical and computational research programs that couple to and advance forefront experimental "tabletop" science directly relevant to DOE. 
FINDING: Progress in the BES theory enterprise requires increased access to the entire spectrum of computational resources contemplated by the Office of Science for the next ten years: (A) BES is ready for and requires access to leadership-scale computing to perform calculations that cannot be done elsewhere. (B) A large amount of essential BES computation falls between the leadership scale and the desktop scale.

RECOMMENDATION: BES should become strongly engaged with the DOE Office of Advanced Scientific Computing Research to ensure that large amounts of time on terascale capacity facilities are available to the BES scientific community. Also, BES should consider supporting some of this capacity with local institutional computing, while ensuring that demand at the higher end of computing power is supplied by larger facilities.

FINDING: The current lack of support for the development and maintenance of shared scientific software diminishes the scientific impact of the BES-supported theory community and creates an obstacle to the effective exploitation of high-end computing resources and facilities.
RECOMMENDATION: BES should support the development and maintenance of scientific codes in the disciplines in its portfolio, just as it now funds the development of shared beamlines at its experimental facilities, thereby creating new scientific capabilities for the nation. Such investments will also be critical in allowing BES researchers to take full advantage of the capabilities of DOE's leadership-class computing facilities.

FINDING: There are not enough theorists or theorists-in-training, and therefore there is an insufficient overall effort devoted to theory and computation to adequately address the needs and opportunities across the broad range of scientific research in BES, as articulated in this report.

RECOMMENDATION: In an expanded program in theory and computation, BES should target its investments in university grants to support the training of students and postdoctoral researchers and to encourage the creation of faculty positions for theoretical and computational researchers. In the DOE laboratories, BES should influence the creation of positions for theorists directly with its investments. 


\section{References}

1. National Nanotechnology Initiative, http://www.nano.gov

2. Thorpe, M. F. and A. E. Carlsson, "The Role of Theory in Biological Physics and Materials: A Report to the National Science Foundation" (2004), http://biophysics.asu.edu/workshop/ report.html.

3. Dixon, D. M., P. T. Cummings, and K. Hess, "Investigative tools: Theory, modeling and simulation," in Nanotechnology Research Directions: IWGN Workshop Report: Vision for Nanotechnolgy in the Next Decacle, M. C. Roco, S. Williams, and P. Alivisatos, editors (Dordrecht: Kluwer Academic Publishers, 2000).

4. Reed, M. A., C. Zhou, C. J. Muller, T. P. Burgin, and J. M. Tour, "Conductance of a molecular junction," Science 278(5336), 252-254 (1997).

5. McCurdy, C. W., E. Stechel, P. T. Cummings, B. Hendrickson, and D. Keyes, Theory and Modeling in Nanoscience: Report of the May 10-11, 2002, Workshop Conducted by the Basic Energy Sciences and Adranced Scientific Conputing Advisory Committees to the Office of Science, Department of Energy, 2002. http://www.sc.doe.gov/bes/reports/list.html

6. Pan, Z. W., S. Dai, D. B. Beach, and D. H. Lowndes, "Temperature dependence of morphologies of aligned silicon oxide nanowire assemblies catalyzed by molten gallium," Nano Letters 3(9), 1279-1284 (2003).

7. Brinker, C. J., Y. Lu, M. Antonietti, and C. Bai, eds., Self-Assembled Nanostmetured Materials (Warrendale, PA: Materials Research Society, 2003).

8. Zhang, S., D. M. Marini, W. Hwang, and S. Santoso, "Design of nanostructured biological materials through self-assembly of peptides and proteins," Current Opinion in Chemical Biology 6(6), 865-871 (2002).
9. Seeman, N. C., and A. M. Belcher, "Emulating biology: Building nanostructures from the bottom up," Proceedings of the National Academy of Sciences of the United States of America 99 (Suppl. 2), 6451-6455 (2002).

10. M. I. Stockman, "Nanofocusing of optical energy in tapered plasmonic waveguides," Phys.

Rev. Lett. 93, 137404 (2004).

11. Cao, D. P., P. Y. Feng, and J. Z. Wu, "Molecular simulation of novel carbonaceous materials for hydrogen storage," Nano Letters 4(8), 1489-1492 (2004).

12. Yarkony, D. R., "Diabolical conical intersections," Reviews of Modern Physics 68, 985-1013 (1996).

13. "Breakthrough of the year: The runners-up," Science 306, 2013 (2004), http://www. sciencemag.org/cgi/content/full/306/5704/ 2013\#condensate.

14. Milosevic, N., P. B. Corkum, and T. Brabec, "How to use lasers for imaging attosecond dynamics of nuclear processes," Phys. Rev. Lett. 92, 013002 (2004).

15. Chelkowski, S., A. D. Bandrauk, and P. B. Corkum, "Muonic molecules in superintense laser fields," Phys. Rev. Lett. 93, 083602 (2004).

16. T. Brabec, private communication.

17. Nitzan, A. and M. A. Ratner, "Electron transport in molecular wire junctions," Science 300, 1384 (2003).

18. Wolf, S. A., D. D. Awschalom, R. A. Buhrman, J. M. Daughton, S. von Molnar, M. L. Roukes, A. Y. Chtchelkanova, and D. M. Treger, "Spintronics: A spin-based electronics vision for the future," Science 294, 1488 (2001).

19. Gimzewski, J. K., and C. Joachim, "Nanoscale science of single molecules using local probes," Science 283, 1683 (1999). 
20. NSF Blue Ribbon Panel on High Performance Computing (Lewis Branscomb, Chairman), "From Desktop to Teraflop: Exploiting the US Lead in High Performance Computing"

(Washington DC: National Science Board NSB 93-205, August 1993).
21. High-End Computing Subcommittee /Gregory McRae, Chairman), "High-Performance Computational Needs and Capabilities in the Office of Science," Advanced Scientific Computing Advisory Committee, Office of Science, U.S. Department of Energy, April 6, 2004. 
Editor: John Hules, Computing Sciences Communications, Lawrence Berkeley National Laboratory

Design, production, and printing: Creative Services Office, Lawrence Berkeley National Laboratory (Alice Ramirez, Flavio Robles, Jr., Michael Muller, Caitlin Youngquist, Faye Jobes, Cheryl Ventimiglia) 


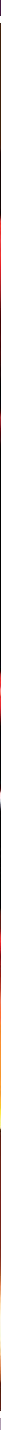

